Randomized, Double-Blind, Placebo-Controlled, Multicenter Phase II Study of Onartuzumab Plus Bevacizumab Versus Placebo Plus Bevacizumab in Patients With Recurrent Glioblastoma: Efficacy, Safety, and Hepatocyte Growth Factor and O(6)-Methylguanine-DNA Methyltransferase Biomarker Analyses

Cloughesy, Timothy ; Finocchiaro, Gaetano ; Belda-Iniesta, Cristóbal ; Recht, Lawrence ; Brandes, Alba A ; Pineda, Estela ; Mikkelsen, Tom ; Chinot, Olivier L ; Balana, Carmen ; Macdonald, David R ;

Westphal, Manfred ; Hopkins, Kirsten ; Weller, Michael ; Bais, Carlos ; Sandmann, Thomas ; Bruey, Jean-Marie ; Koeppen, Hartmut ; Liu, Bo ; Verret, Wendy ; Phan, See-Chun ; Shames, David S

DOI: https://doi.org/10.1200/JCO.2015.64.7685

Posted at the Zurich Open Repository and Archive, University of Zurich ZORA URL: https://doi.org/10.5167/uzh-141041

Journal Article

Published Version

Originally published at:

Cloughesy, Timothy; Finocchiaro, Gaetano; Belda-Iniesta, Cristóbal; Recht, Lawrence; Brandes, Alba A; Pineda, Estela; Mikkelsen, Tom; Chinot, Olivier L; Balana, Carmen; Macdonald, David R; Westphal, Manfred; Hopkins, Kirsten; Weller, Michael; Bais, Carlos; Sandmann, Thomas; Bruey, Jean-Marie; Koeppen, Hartmut; Liu, Bo; Verret, Wendy; Phan, See-Chun; Shames, David S (2017). Randomized, Double-Blind, Placebo-Controlled, Multicenter Phase II Study of Onartuzumab Plus Bevacizumab Versus Placebo Plus Bevacizumab in Patients With Recurrent Glioblastoma: Efficacy, Safety, and Hepatocyte Growth Factor and O(6)-Methylguanine-DNA Methyltransferase Biomarker Analyses. Journal of Clinical Oncology, 35(3):343-351.

DOI: https://doi.org/10.1200/JCO.2015.64.7685 
Author affiliations appear at the end of this article.

Published at ascopubs.org/journal/jco on December 5, 2016

Support information appears at the end of this article.

Previously presented in part at the 19th Annual Scientific Meeting and Education Day of the Society for Neuro-Oncology, Miami, FL, November 13-16, 2014, and the 2015 ASCO Annual Meeting, Chicago, IL, May 29 to June 2, 2015.

Clinical trial information: NCT01632228.

Corresponding author: Timothy

Cloughesy, MD, UCLA Neuro-Oncology Program, 710 Westwood Plaza, Suite

1-230, Los Angeles, CA 90095; e-mail: tcloughesy@mednet.ucla.edu.

C 2016 by American Society of Clinical Oncology

0732-183X/17/3503w-343w/\$20.00

\section{Randomized, Double-Blind, Placebo-Controlled, Multicenter Phase II Study of Onartuzumab Plus Bevacizumab Versus Placebo Plus Bevacizumab in Patients With Recurrent Glioblastoma: Efficacy, Safety, and Hepatocyte Growth Factor and $\mathrm{O}^{6}$-Methylguanine-DNA Methyltransferase Biomarker Analyses}

Timothy Cloughesy, Gaetano Finocchiaro, Cristóbal Belda-Iniesta, Lawrence Recht, Alba A. Brandes, Estela Pineda, Tom Mikkelsen, Olivier L. Chinot, Carmen Balana, David R. Macdonald, Manfred Westphal, Kirsten Hopkins, Michael Weller, Carlos Bais, Thomas Sandmann, Jean-Marie Bruey, Hartmut Koeppen, Bo Liu, Wendy Verret, See-Chun Phan, and David S. Shames

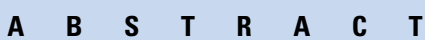

\section{Purpose}

Bevacizumab regimens are approved for the treatment of recurrent glioblastoma in many countries. Aberrant mesenchymal-epithelial transition factor (MET) expression has been reported in glioblastoma and may contribute to bevacizumab resistance. The phase II study GO27819 investigated the monovalent MET inhibitor onartuzumab plus bevacizumab (Ona + Bev) versus placebo plus bevacizumab (Pla + Bev) in recurrent glioblastoma.

\section{Methods}

At first recurrence after chemoradiation, bevacizumab-naïve patients with glioblastoma were randomly assigned 1:1 to receive Ona $(15 \mathrm{mg} / \mathrm{kg}$, once every 3 weeks) + Bev (15 mg/kg, once every 3 weeks) or Pla + Bev until disease progression. The primary end point was progression-free survival by response assessment in neuro-oncology criteria. Secondary end points were overall survival, objective response rate, duration of response, and safety. Exploratory biomarker analyses correlated efficacy with expression levels of MET ligand hepatocyte growth factor, $\mathrm{O}^{6}$-methylguanine-DNA methyltransferase promoter methylation, and glioblastoma subtype.

\section{Results}

Among 129 patients enrolled (Ona + Bev, $n=64$; Pla + Bev, $n=65$ ), baseline characteristics were balanced. The median progression-free survival was 3.9 months for Ona + Bev versus 2.9 months for $\mathrm{Pla}+\mathrm{Bev}$ (hazard ratio, 1.06; $95 \% \mathrm{Cl}, 0.72$ to $1.56 ; P=.7444$ ). The median overall survival was 8.8 months for Ona + Bev and 12.6 months for Pla + Bev (hazard ratio, 1.45; 95\% Cl, 0.88 to 2.37; $P=$.1389). Grade $\geq 3$ adverse events were reported in $38.5 \%$ of patients who received Ona + Bev and $35.9 \%$ of patients who received $\mathrm{Pla}+$ Bev. Exploratory biomarker analyses suggested that patients with high expression of hepatocyte growth factor or unmethylated $\mathrm{O}^{6}$-methylguanine-DNA methyltransferase may benefit from Ona + Bev.

\section{Conclusion}

There was no evidence of further clinical benefit with the addition of onartuzumab to bevacizumab compared with bevacizumab plus placebo in unselected patients with recurrent glioblastoma in this phase II study; however, further investigation into biomarker subgroups is warranted.

\section{J Clin Oncol 35:343-351. (C) 2016 by American Society of Clinical Oncology}

\section{INTRODUCTION}

Standard treatment of newly diagnosed glioblastoma (the most common primary tumor in the $\mathrm{CNS}^{1,2}$ ) is surgical debulking followed by radiotherapy and temozolomide, providing a median overall survival (OS) of 14.6 months. ${ }^{3,4}$ Recent data suggest that glioblastoma comprises clinically relevant subtypes (proneural, mesenchymal, and proliferative/neural) that exhibit different prognostic outcomes and treatment 
responses. ${ }^{5}$ Prognostic benefits have been reported for mesenchymal and neural subtypes, unlike proneural subtypes. ${ }^{5}$

Glioblastomas are highly vascularized tumors ${ }^{6}$ and respond to antiangiogenic agents. ${ }^{7-13}$ The Avastin in Glioblastoma (AVAglio) and RTOG-0825 studies evaluated first-line radiotherapy, temozolomide, and either bevacizumab or placebo in newly diagnosed gliomas; AVAglio reported median progression-free survival (PFS) of 10.6 versus 6.2 months, respectively, ${ }^{14}$ and RTOG-0825 reported median PFS of 10.7 versus 7.3 months, respectively. ${ }^{15}$ However, neither study demonstrated an OS benefit for the bevacizumabcontaining regimen. A retrospective subgroup analysis of AVAglio reported that mesenchymal and proneural subtypes derived PFS benefit with the bevacizumab combination; however, only the proneural subtype reported a survival benefit. ${ }^{16}$

The mesenchymal-epithelial transition factor (MET) pathway can promote proliferation, survival, and metastasis in tumors and can be dysregulated by MET receptor mutations or amplification, and overexpression of its ligand, hepatocyte growth factor (HGF). ${ }^{17}$ Murine models suggest that the MET pathway may lead to resistance in glioblastomas receiving antiangiogenic therapy. ${ }^{18}$ Glioblastomas can express MET as assessed by immunohistochemistry $(\mathrm{IHC})^{19,20}$; approximately $88 \%$ have aberrant downstream MET signaling, and $4 \%$ have MET amplification. ${ }^{21}$ Expression of HGF is also seen in glioblastomas. ${ }^{21,22}$ Onartuzumab, a humanized, monovalent monoclonal anti-MET antibody, demonstrated inhibition of glioblastoma growth in preclinical testing. ${ }^{23}$ Therefore, the MET pathway is a rational target to investigate in glioblastoma. The phase II GO27819 study (NCT01632228) investigated the safety and efficacy of onartuzumab plus bevacizumab versus placebo plus bevacizumab in recurrent glioblastoma.

\section{PATIENTS AND METHODS}

At first recurrence after chemoradiation, bevacizumab-naïve patients with glioblastoma were randomly assigned 1:1 by interactive voice/Web response system to receive intravenous onartuzumab $(15 \mathrm{mg} / \mathrm{kg})$ plus bevacizumab $(15 \mathrm{mg} / \mathrm{kg}$; Ona $+\mathrm{Bev})$ or placebo plus bevacizumab (Pla + Bev) in 3-weekly cycles (day 1) until disease progression. Enrollment in a Pla + Ona arm was put on hold during an initial safety assessment and was subsequently closed as a result of concerns regarding unequal randomization. Patients were stratified by age ( $<50$ years $v \geq 50$ years) and Karnofsky performance status ( $70 \%$ to $80 \% v 90 \%$ to $100 \%$ ).

To meet inclusion criteria, patients had to be at least 18 years old with Karnofsky performance status $\geq 70 \%$; with histologically confirmed glioblastoma at first recurrence (by Response Assessment in NeuroOncology criteria ${ }^{24}$ ) after concurrent or adjuvant chemoradiotherapy (no more than one previous temozolomide-based regimen; no previous therapy targeting angiogenic or MET pathways). Prior therapy with Gamma Knife or focal high-dose radiotherapy was allowed if histologic recurrence was documented.

Patients were excluded if they were unable to undergo brain magnetic resonance imaging scans with intravenous gadolinium. Patients with absolute neutrophil count $<1.5 \times 10^{9} / \mathrm{L}$, platelet count $<100 \times 10^{9} / \mathrm{L}$, hemoglobin $<9.0 \mathrm{~g} / \mathrm{dL}$, total bilirubin $\geq 1.5 \times$ upper limit of normal, serum creatinine $>1.5 \times$ upper limit of normal, calculated creatinine clearance $<60 \mathrm{~mL} / \mathrm{min}$, or urine dip-stick test for proteinuria $\geq 2+$ were also excluded. Patients who had prior treatment with prolifeprospan 20 carmustine wafer or a prior intracerebral agent were excluded. Patients with inadequately controlled hypertension or diabetes, history of hypertensive crisis, hypertensive encephalopathy, or significant vascular disease were not included. Patients with evidence of bleeding diathesis or coagulopathy, history of abdominal fistula, GI perforation or intracranial abscess within 6 months before random assignment, or history of another malignancy in the previous 3 years (with a disease-free interval of $<3$ years) were also excluded, as were those with serious nonhealing wounds, active ulcers, or untreated bone fractures.

Paraffin-embedded tumor samples were required to determine MET IHC expression status. ${ }^{25}$ Tissue from recurrent surgery was preferred, but tissue from the initial surgery was sufficient for study entry. MET-positive status was initially defined as $\geq 50 \%$ of tumor cells with membrane and/or cytoplasmic staining at moderate-to-high intensity. An exploratory METpositive status cutoff of $\geq 10 \%$ was also evaluated.

HGF RNA expression was assessed by cobas polymerase chain reaction (PCR; Roche Molecular Systems, Pleasanton, CA), with high expression classed as the upper $25 \%$ subgroup and low expression as the lower $75 \%$ subgroup on the basis of analysis of the Subpopulation Treatment Effect Pattern Plots (Appendix Fig A1, online only). HGF expression was also evaluated by nonisotopic in situ hybridization (ISH) using branched DNA technology (Advanced Cell Diagnostics, Hayward, CA; Appendix Fig A2, online only). Promoter methylation of DNA repair gene $\mathrm{O}^{6}$-methylguanine-DNA methyltransferase (MGMT), a potential positive prognostic marker for glioblastomas, ${ }^{26}$ was also evaluated by quantitative methylation-specific PCR. ${ }^{27}$ Retrospective analyses of AVAglio indicated that glioblastoma subtypes were differentially associated with bevacizumab efficacy ${ }^{16}$; therefore, in this analysis, correlation between glioblastoma subtype determined by NanoString gene expression and efficacy was assessed (Appendix, online only).

The coprimary end points were PFS by Response Assessment in NeuroOncology criteria in the intent-to-treat (ITT) population and the METpositive subpopulation. Secondary end points (for ITT and MET biomarker-positive subgroups) included median OS, 9-month OS rate, 6month PFS rate, overall response rate (ORR), duration of response, and safety. PFS and OS were assessed using the Kaplan-Meier method. $P$ values of Ona + $\mathrm{Bev}$ versus $\mathrm{Pla}+\mathrm{Bev}$ were obtained from either the log-rank test or the Cox model, and hazard ratios (HRs) were estimated from the Cox model. The biomarker effect was further evaluated using the multivariable Cox model, including the treatment, biomarker, their interaction, and baseline characteristics. Statistical significance was set at .05 (two-sided significance). Safety was assessed per National Cancer Institute Common Terminology Criteria for Adverse Events version 4.0. The study was conducted in 42 centers across eight countries, in accordance with the Declaration of Helsinki and Good Clinical Practice principles; patients provided written informed consent.

\section{RESULTS}

\section{Patients}

Between June 2012 and February 2013, 64 patients were randomly assigned to Ona $+\mathrm{Bev}$ and 65 to $\mathrm{Pla}+\mathrm{Bev}$. Patient disposition is shown in Fig 1. The clinical data cutoff was November 7, 2013. The median follow-up was 9.8 and 9.9 months, respectively, for Ona $+\mathrm{Bev}$ and $\mathrm{Pla}+\mathrm{Bev}$. At the clinical cutoff, 59 patients receiving Ona $+\mathrm{Bev}(92 \%)$ and 55 patients receiving $\mathrm{Pla}+$ Bev (85\%) had stopped study treatment (Fig 1).

Baseline characteristics were balanced between the arms (Table 1). Most patients (91.5\%) had tumors with low MET expression (MET IHC score 0, 1+; IHC 50\% staining cutoff). For the $10 \%$ staining cutoff, $79.8 \%$ of tumors were MET negative. Because only five patients across the arms had MET-positive tumors by the $50 \%$ staining cutoff, this sample was deemed too small to produce meaningful results; therefore, the planned analyses in this subgroup were cancelled. 


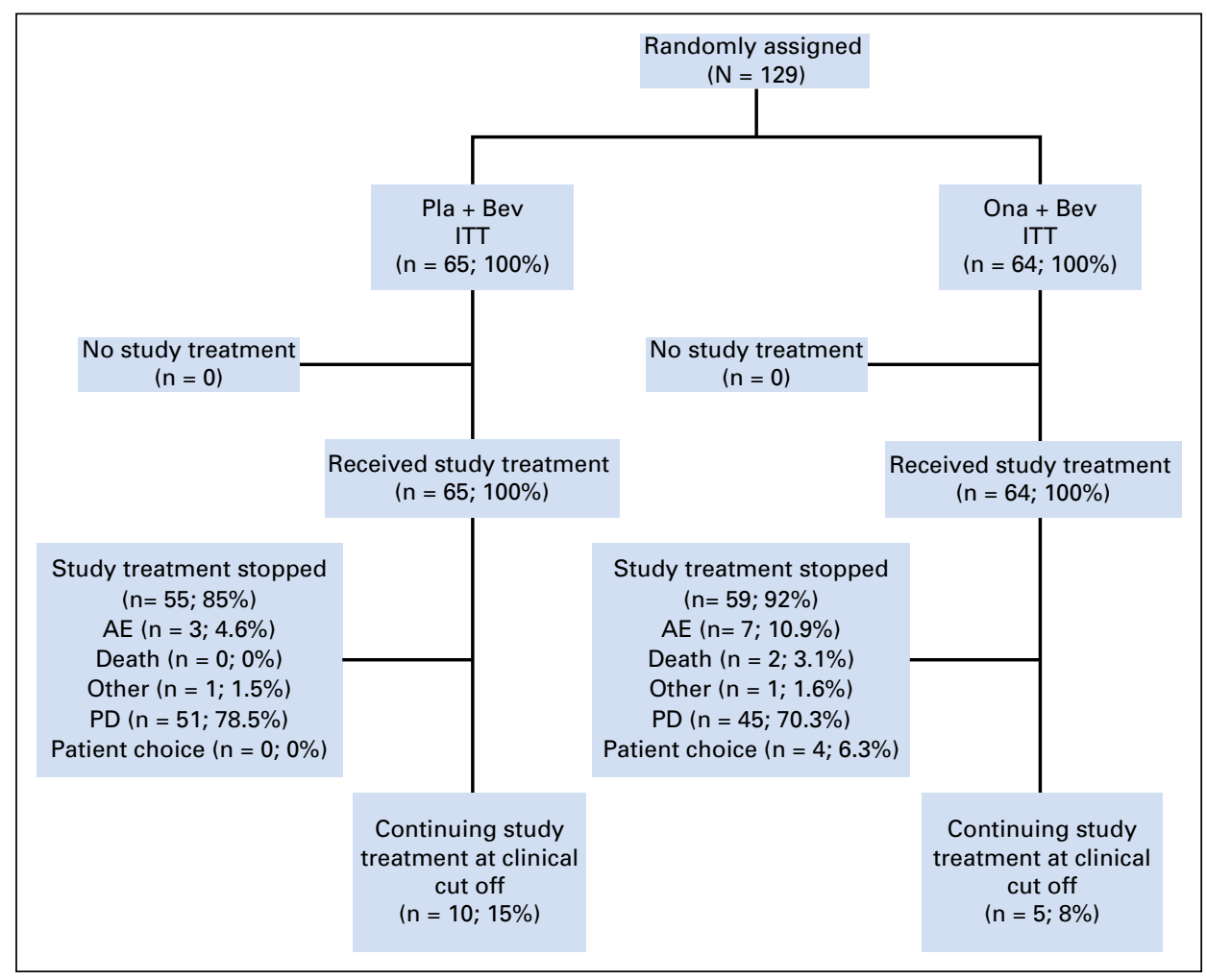

Fig 1. CONSORT diagram. Patient disposition. $A E$, adverse event; ITT, intent to treat; Ona + Bev, onartuzumab plus bevacizumab; $\mathrm{PD}$, progressive disease; $\mathrm{Pla}+\mathrm{Bev}$, placebo plus bevacizumab.

\section{Efficacy}

The median PFS (ITT) was 3.9 months for Ona + Bev and 2.9 months for $\mathrm{Pla}+\mathrm{Bev}(\mathrm{HR}, 1.06 ; 95 \% \mathrm{CI}, 0.72$ to 1.56 ; $P=.7444$; Fig 2A). Subgroup analyses of PFS were consistent with ITT data (Fig 2B). The median OS was 8.8 months for Ona + Bev and 12.6 months for Pla $+\mathrm{Bev}$ (HR, 1.45; 95\% CI, 0.88 to 2.37; $P=.1389$; Fig 2C). Predefined subgroup analyses of OS were generally consistent with ITT results (Fig 2D). The 6-month PFS rate was 33.9\% for Ona $+\mathrm{Bev}$ and $29.0 \%$ for $\mathrm{Pla}+\mathrm{Bev}(P=.5555)$. The 9-month OS rates were $49.7 \%$ and $57.2 \%$, respectively, for Ona $+\mathrm{Bev}$ and $\mathrm{Pla}+\mathrm{Bev}(P=.4115)$. The ORR was $22.2 \%$ in the Ona + Bev arm (12 of 54 patients; one complete response) and $23.7 \%$ in the $\mathrm{Pla}+\mathrm{Bev}$ arm (14 of 59 patients; three complete responses). The median duration of response was 6.4 months for Ona + Bev and 9.7 months for Pla + Bev.

\section{Exploratory Biomarker Analysis}

Glioblastoma subtype. Recent retrospective analysis from AVAglio indicated that glioblastoma subtypes were differentially associated with outcomes to bevacizumab ${ }^{16}$; therefore, glioblastoma subtype was evaluated in GO27819. There were proportionally more mesenchymal-subtype tumors (47\% v 41\%, respectively) and fewer proneural tumors $(23.5 \% \vee 30.3 \%$, respectively) in the GO27819 study than in AVAglio. The results showed that there was no difference in PFS between the mesenchymal subtype compared with other subtypes in GO27819 (Appendix Fig A3, online only).

Because MET/HGF signaling is essential in maintaining the mesenchymal phenotype, the association between HGF expression and glioblastoma subtype was analyzed in AVAglio and GO27819 using NanoString technology. ${ }^{16}$ As expected, HGF expression was associated with the mesenchymal phenotype in both studies, suggesting that this may be a common occurrence in glioblastoma biology (Appendix Tables A1 to A3, online only).

\section{HGF Analysis}

Analysis of $H G F$ expression in the AVAglio study showed no significant differences in PFS (HR, 1.25; $P=.24$ ) or OS (HR, 1.11; $P=.57)$ for high expression of $H G F$ versus low expression of $H G F$ in the control arm; however, low expression of HGF seemed to be predictive of improved efficacy for bevacizumab (PFS: HR, 1.71; $P=.0056$; OS: HR, 1.56; $P=.027$; Appendix Fig A4, online only). Therefore, it was decided to correlate HGF expression with efficacy in GO27819.

In GO27819, 119 patients (Ona $+\mathrm{Bev}, \mathrm{n}=58$; Pla $+\mathrm{Bev}$, $\mathrm{n}=61$ ) had PCR results for HGF available. In the Pla $+\mathrm{Bev}$ arm, patients with high HGF expression (upper $25 \%, \mathrm{n}=16$ ) had shorter efficacy outcomes than those with lower PCR levels of HGF (lower 75\%, $\mathrm{n}=45$ ) for PFS (median PFS: $2.8 \vee 4.1$ months, respectively; $\mathrm{HR}, 1.67 ; 95 \% \mathrm{CI}, 0.90$ to $3.13 ; P=.1167$ ) and $\mathrm{OS}$ (median OS: 7.3 months for the upper 25\% group and not reached [NR] for the lower $75 \%$ group; HR, 1.65 ; $95 \%$ CI, 0.76 to 3.59 ; $P=.2012$ ); however, the differences were not statistically significant.

In patients with PCR expression of HGF in the upper $25 \%$ subgroup (Ona $+\mathrm{Bev}, \mathrm{n}=14 ; \mathrm{Pla}+\mathrm{Bev}, \mathrm{n}=16$ ), significantly longer median PFS was seen with Ona + Bev (6.1 months) versus Pla + Bev (2.8 months; HR, 0.37 ; $95 \%$ CI, 0.16 to $0.86 ; P=.0201$; Fig $3 \mathrm{~A}$ ). The median OS was longer with Ona + Bev in this subgroup (NR for Ona + Bev $v 7.3$ months for Pla + Bev; HR, 0.29; 95\% CI, 0.08 to $1.06 ; P=.0604$; Fig $3 B$ ). Patients in the upper $25 \%$ subgroup of PCR 


\begin{tabular}{|c|c|c|}
\hline Characteristic & $\begin{array}{c}\text { Ona }+ \text { Bev } \\
(n=64)\end{array}$ & $\begin{array}{c}\mathrm{Pla}+\mathrm{Bev} \\
(\mathrm{n}=65)\end{array}$ \\
\hline Median age, years & 57.0 & 55.0 \\
\hline Age $<50$ years & $18(28.1)$ & $17(26.2)$ \\
\hline Age $\geq 50$ years & 46 (71.9) & 48 (73.8) \\
\hline \multicolumn{3}{|l|}{ Sex } \\
\hline Male & $44(68.8)$ & $39(60.0)$ \\
\hline Female & 20 (31.3) & $26(40.0)$ \\
\hline \multicolumn{3}{|l|}{ Karnofsky performance status } \\
\hline $70 \%-80 \%$ & $36(56.3)$ & 37 (56.9) \\
\hline $90 \%-100 \%$ & 28 (43.8) & $28(43.1)$ \\
\hline \multicolumn{3}{|l|}{ Race } \\
\hline Asian & $3(4.7)$ & $0(0.0)$ \\
\hline White & 59 (92.2) & 64 (98.5) \\
\hline Other & $2(3.1)$ & $0(0.0)$ \\
\hline Multiple & $0(0.0)$ & $1(1.5)$ \\
\hline Corticosteroid use at baseline & $10(15.6)$ & $15(23.1)$ \\
\hline \multicolumn{3}{|l|}{ Prior surgery } \\
\hline Biopsy & $5(7.8)$ & $2(3.1)$ \\
\hline Complete resection & $32(50.0)$ & 38 (58.5) \\
\hline Partial resection & $26(40.6)$ & $23(35.4)$ \\
\hline Measurable disease at baseline* & $54(84.4)$ & 59 (90.8) \\
\hline \multicolumn{3}{|l|}{ Number of target lesions at baseline } \\
\hline$\leq 1$ & 39 (60.9) & $47(72.3)$ \\
\hline$>1$ & $15(23.4)$ & $12(18.5)$ \\
\hline Glioblastoma subtype & $n=58$ & $n=61$ \\
\hline Mesenchymal & $27(46.6)$ & $29(47.5)$ \\
\hline Proneural & $14(24.1)$ & $14(23.0)$ \\
\hline Proliferative & $10(17.2)$ & 10 (16.4) \\
\hline Unclassified & $7(12.1)$ & $8(13.1)$ \\
\hline \multicolumn{3}{|c|}{ MET IHC score (50\% staining cutoff) } \\
\hline 0 & $52(81.3)$ & 53 (81.5) \\
\hline $1+$ & $4(6.3)$ & $9(13.8)$ \\
\hline $2+$ & $3(4.7)$ & $1(1.5)$ \\
\hline $3+$ & $0(0.0)$ & $1(1.5)$ \\
\hline \multicolumn{3}{|l|}{ MET IHC (10\% staining cutoff) } \\
\hline 0 & $35(54.7)$ & 35 (53.8) \\
\hline $1+$ & $17(26.6)$ & $16(24.6)$ \\
\hline $2+$ & $7(10.9)$ & 11 (16.9) \\
\hline $3+$ & $0(0.0)$ & $2(3.1)$ \\
\hline HGF PCR status & $\mathrm{n}=58$ & $\mathrm{n}=61$ \\
\hline Upper 25\% & $14(24.1)$ & $16(26.2)$ \\
\hline Lower 75\% & 44 (75.9) & 45 (73.8) \\
\hline \multicolumn{3}{|l|}{ HGF ISH score } \\
\hline 0 & $16(25.0)$ & $17(26.2)$ \\
\hline $1+$ & 21 (32.8) & $25(38.5)$ \\
\hline $2+$ & $16(25.0)$ & $18(27.7)$ \\
\hline $3+$ & $4(6.3)$ & $3(4.6)$ \\
\hline MGMT methylation status & $n=56$ & $n=54$ \\
\hline Methylated & $21(37.5)$ & $26(48.1)$ \\
\hline Unmethylated & $32(57.1)$ & $25(46.3)$ \\
\hline Possible, weak methylation & $3(5.4)$ & $3(5.6)$ \\
\hline IDH1 mutation status & $n=58$ & $n=57$ \\
\hline Mutation-positive & $4(6.9)$ & $5(8.8)$ \\
\hline Wild-type & $54(93.1)$ & $57(91.2)$ \\
\hline \multicolumn{3}{|c|}{$\begin{array}{l}\text { NOTE. Data presented as No. (\%) unless otherwise noted. } \\
\text { Abbreviations: Bev, bevacizumab; } H G F \text {, hepatocyte growth factor; IDH1, iso } \\
\text { citrate dehydrogenase } 1 ; \text {; IHC, immunohistochemistry; ISH, in situ hybridization } \\
\text { MET, mesenchymal-epithelial transition factor; MGMT, O6-methylguanine-DNA } \\
\text { methyltransferase; Ona, onartuzumab; PCR, polymerase chain reaction; Pla } \\
\text { placebo. } \\
{ }^{*} \text { Confirmation of measurable disease was not a protocol-defined inclusion } \\
\text { criterion. }\end{array}$} \\
\hline
\end{tabular}

expression of $H G F$ also had significantly higher ORR with Ona + Bev compared with $\mathrm{Pla}+\mathrm{Bev}(35.7 \% v 0 \% ; P=.014)$.

Among patients with PCR expression of HGF in the lower $75 \%$ subgroup, those treated with Ona $+\operatorname{Bev}(n=44)$ had shorter median PFS (2.8 $v$ 4.1 months, respectively; HR, 1.39; 95\% CI, 0.87 to $2.20 ; P=.1589)$ and significantly shorter OS (8.6 months $v$ NR, respectively; HR, $1.86 ; 95 \% \mathrm{CI}, 1.03$ to $3.36 ; P=.0381$ ) compared with those treated with Pla $+\mathrm{Bev}(\mathrm{n}=45$; Fig $3 \mathrm{C}$ and $\mathrm{D})$.

HGF expression was also assessed by ISH in GO27819 and correlated with PFS and OS results (Appendix Fig A5, online only). The overlap of high versus low HGF expression as assessed by PCR versus ISH is shown in Appendix Table A4 (online only).

Although the mesenchymal subtype did not predict outcomes, $H G F$ levels seemed to be predictive of Ona + Bev efficacy. To evaluate this, we compared outcomes for the mesenchymal subtype or other subtypes stratified by HGF expression (Appendix Fig A6, online only). There was no difference in outcomes between the arms for the mesenchymal subtype with low HGF expression, whereas patients with high $H G F$ expression seemed to derive benefit from the addition of onartuzumab to bevacizumab. There were no differences in outcomes for patients in the nonmesenchymal subset with low HGF expression. The nonmesenchymal subset of patients with high HGF expression was too small $(\mathrm{n}=9)$ to robustly evaluate efficacy.

\section{MGMT Methylation Analysis}

Methylation of $M G M T$ is a well-known prognostic marker in glioblastoma. Thus 110 patients (Ona + Bev, $\mathrm{n}=56$; Pla $+\mathrm{Bev}$, $\mathrm{n}=54)$ were analyzed for MGMT methylation status. Forty-seven patients had methylated MGMT, 57 had unmethylated MGMT, and six patients had unconfirmed MGMT status. Assessing the Pla + Bev arm only, patients had a worse outcome when treated with $\mathrm{Pla}+\mathrm{Bev}$ if they had unmethylated MGMT and a better prognosis if they had methylated MGMT (PFS: HR, 3.19; 95\% CI, 1.58 to 6.44; OS: HR, 3.39; 95\% CI, 1.32 to 8.75; Appendix Fig A7, online only).

Median PFS and OS were longer with Ona $+\operatorname{Bev}(n=32)$ compared with $\mathrm{Pla}+\mathrm{Bev}(\mathrm{n}=25)$ in patients with unmethylated MGMT (Fig 4); the median PFS was $4.2 v 2.8$ months, respectively (HR, $0.46 ; 95 \% \mathrm{CI}, 0.25$ to $0.84 ; P=.0108$ ) and the median OS was $10.9 \vee 7.5$ months, respectively (HR, $0.53 ; 95 \% \mathrm{CI}, 0.26$ to $1.10 ; P=.0836)$. A numerically higher ORR was seen with Ona + Bev compared with $\mathrm{Pla}+\mathrm{Bev}$ in the unmethylated MGMT subgroup $(15.6 \% \vee 8.0 \% ; P=.450)$. Among patients with methylated MGMT, those treated with Ona $+\mathrm{Bev}(\mathrm{n}=21)$ had shorter median PFS compared with patients treated with Pla + $\operatorname{Bev}(\mathrm{n}=26 ; 2.8 v 6.4$ months, respectively; HR, $1.52 ; 95 \% \mathrm{CI}, 0.75$ to 3.08; $P=.2440)$ and significantly shorter median OS (7.7 months $v$ NR, respectively; HR, 3.18; 95\% CI, 1.19 to $8.51 ; P=.0150$ ).

Results of multivariable analyses of the HGF and MGMT biomarkers are shown in Appendix Table A5 (online only). With adjustment by the baseline characteristics, MGMT status and PCR analysis of HGF still result in statistically significant effects for PFS and OS by MGMT methylation, and PFS by high or low HGF expression. There was also a potentially predictive treatment effect of Ona + Bev for patients with unmethylated MGMT or with high HGF expression by PCR. The multivariable results were generally consistent with the subgroup analyses.

Correlation between MGMT methylation and PFS benefit with bevacizumab in AVAglio was also investigated. No predictive influence of MGMT methylation status on PFS benefit 


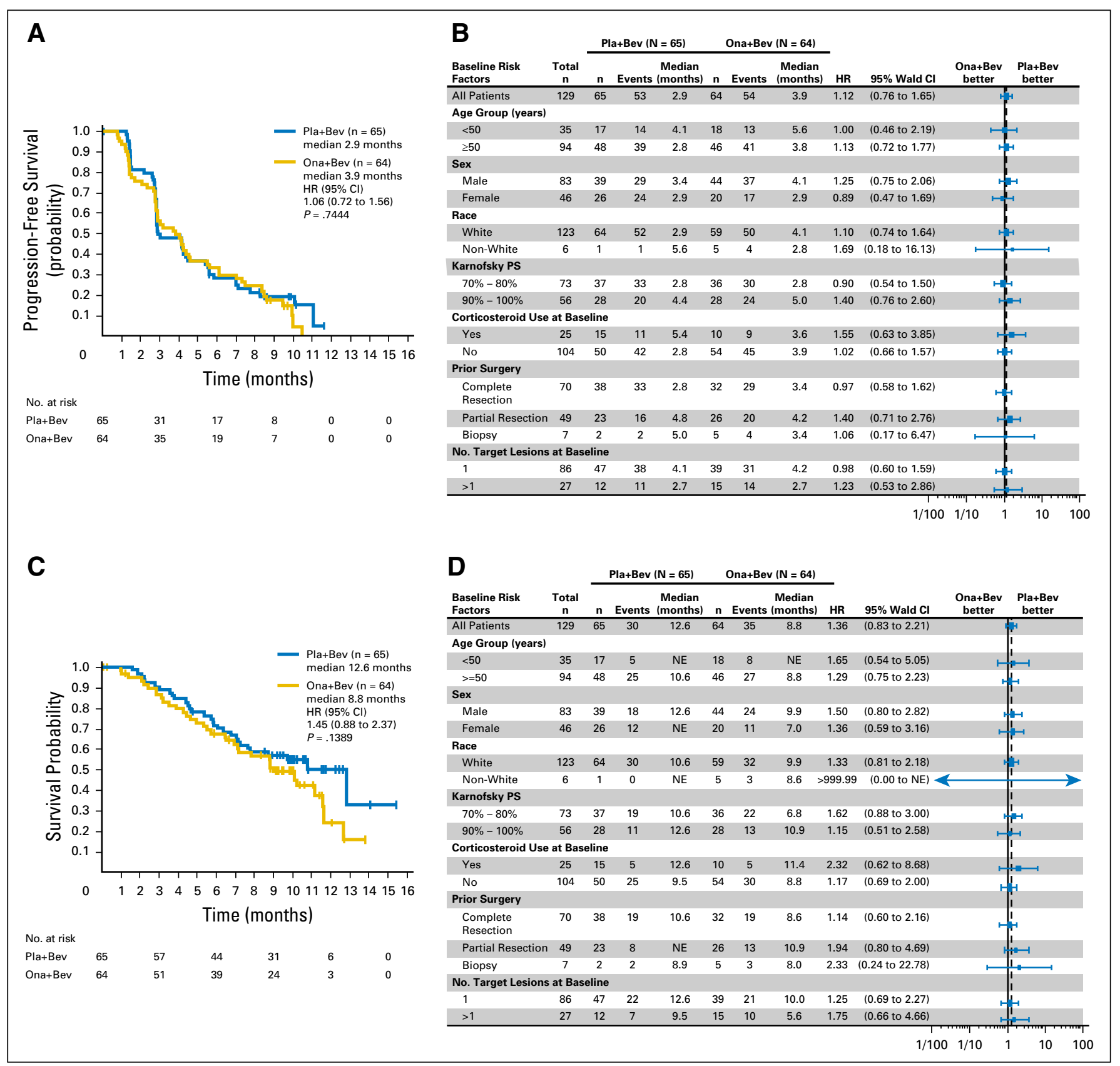

Fig 2. (A) Progression-free survival in the intent-to-treat population and (B) forest plot of progression-free survival subgroup analysis; (C) overall survival in the intent-totreat population and (D) forest plot of overall survival subgroup analysis. HR, hazard ratio; NE, non-evaluable; Ona + Bev, onartuzumab plus bevacizumab; Pla + Bev, placebo plus bevacizumab; PS, performance status.

with bevacizumab was observed (HR, 0.76 for methylated; HR, 0.56 for unmethylated). ${ }^{14}$

\section{Safety}

The median number of cycles for bevacizumab was five for Ona + Bev and six for Pla + Bev; the median number of cycles for onartuzumab was four and six for placebo. The most common classes of adverse events (AEs; any grade) were general disorders $(70.8 \%$ Ona $+\mathrm{Bev} v 57.8 \% \mathrm{Pla}+\mathrm{Bev})$, including peripheral edema $(44.6 \% v 14.1 \%)$, asthenia $(24.6 \% v 21.9 \%)$, and fatigue $(15.4 \% v$
20.3\%); and nervous system disorders (49.2\% Ona $+\operatorname{Bev} v 75.0 \%$ $\mathrm{Pla}+\mathrm{Bev})$, including headaches $(15.4 \% v 23.4 \%)$. Grade $\geq 3$ AEs were reported in $38.5 \%(\mathrm{n}=25)$ of patients receiving Ona $+\mathrm{Bev}$ and $35.9 \%(\mathrm{n}=23)$ of those receiving $\mathrm{Pla}+\mathrm{Bev}$, including nervous system disorders $(7.7 \% v 18.8 \%)$, vascular disorders $(4.6 \% v$ $6.3 \%)$, and respiratory disorders ( $4.6 \% v 1.6 \%)$. Serious AEs were reported in $30.8 \%$ and $29.7 \%$ of patients, respectively, for Ona + Bev and Pla + Bev (Table 2). Grade 5 AEs were reported for two patients receiving Ona $+\mathrm{Bev}$ (two cases of intestinal perforation) and one patient receiving $\mathrm{Pla}+\mathrm{Bev}$ (intracranial hemorrhage). AEs (all grades) with a difference in incidence $\geq 10 \%$ between the arms 

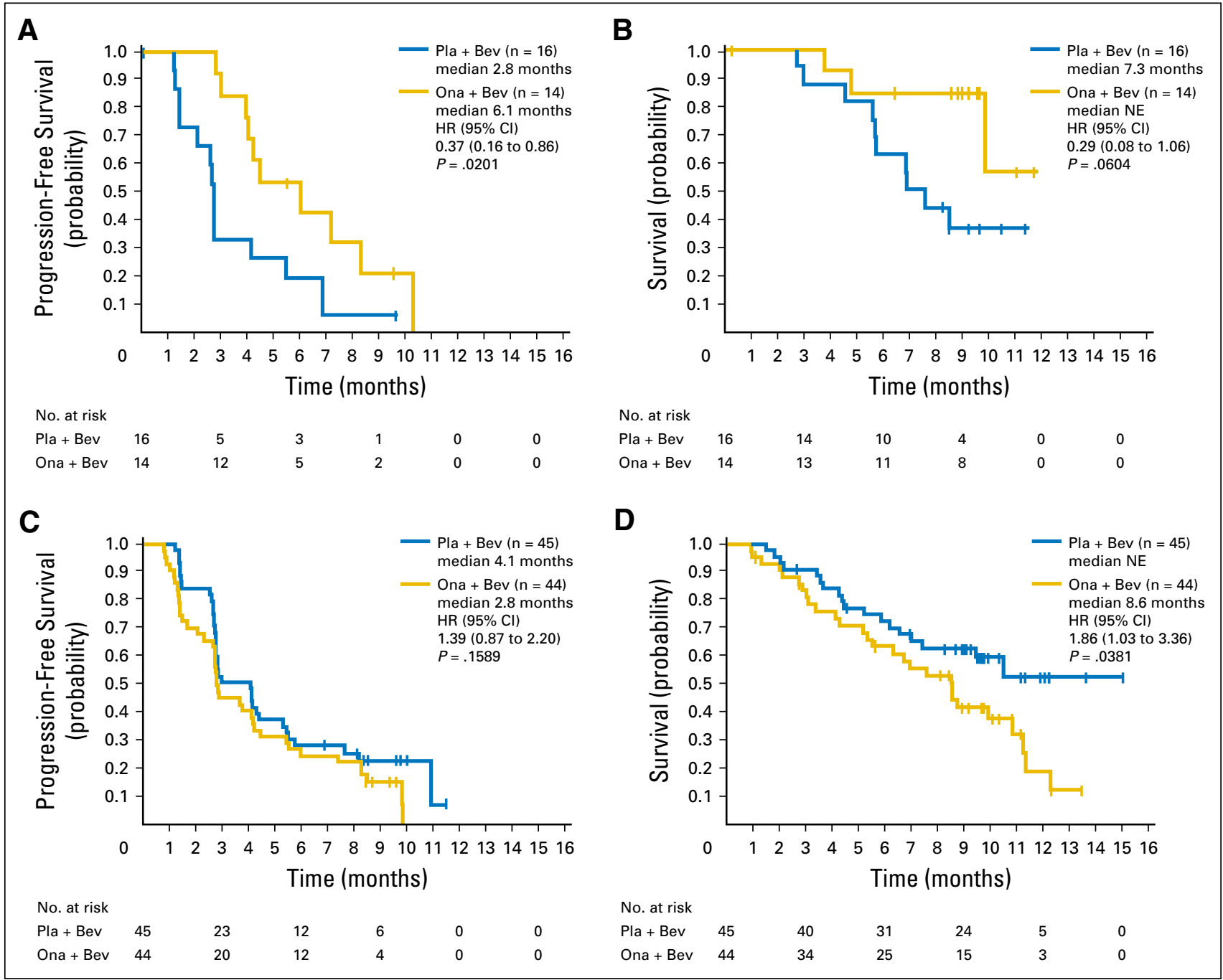

$\begin{array}{lllllll}\text { No. at risk } & & & & & & \\ \text { Pla }+ \text { Bev } & 45 & 23 & 12 & 6 & 0 & 0 \\ \text { Ona }+ \text { Bev } & 44 & 20 & 12 & 4 & 0 & 0\end{array}$

Fig 3. (A) Progression-free survival and (B) overall survival in the polymerase chain reaction levels of hepatocyte growth factor in the upper $25 \%$ subgroup; (C)

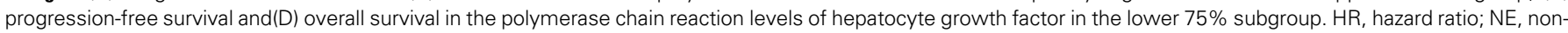
evaluable; Ona + Bev, onartuzumab plus bevacizumab; Pla + Bev, placebo plus bevacizumab.

were peripheral edema (44.6\% Ona + Bev $v$ 14.1\% Pla + Bev), hypertension (12.3\% Ona + Bev $v 32.8 \% \mathrm{Pla}+\mathrm{Bev})$, and hypoalbuminemia (12.3\% Ona + Bev $v 1.6 \% \mathrm{Pla}+\mathrm{Bev})$.

\section{DISCUSSION}

Because recurrent glioblastoma has no standard treatment, ${ }^{28}$ novel treatment combinations are continually being investigated. GO27819 evaluated the antiangiogenic agent bevacizumab plus the anti-MET antibody onartuzumab in recurrent glioblastoma.

The GO27819 study reported a similar median PFS for Ona + $\mathrm{Bev}$ relative to the $\mathrm{Pla}+\mathrm{Bev}$ control. The median PFS with Ona + Bev (3.9 months) was similar to that observed with bevacizumab plus lomustine (4.0 months) in the BELOB study. ${ }^{12}$ The phase II BRAIN study of bevacizumab plus irinotecan reported slightly longer median PFS (5.6 months for bevacizumab plus irinotecan $v$ 4.2 months for bevacizumab alone $)^{7}$ than that reported in GO27819. A median PFS of 3.6 months and a 6-month PFS rate of
$18.8 \%$ was reported for a combination of bevacizumab with temozolomide. ${ }^{13}$ Therefore, the PFS results in GO27819 are in the range of those previously reported for bevacizumab-based combination regimens for treatment of recurrent glioblastoma. However, it is important to note that cross-trial comparisons should be evaluated with caution, because there may be differences in patient populations and neuroimaging assessment methods.

A planned efficacy analysis by MET IHC status could not be performed because of the low prevalence of MET-positive tumors, a limitation of this study. This could have been avoided if the study had been designed to enrich for MET-positive tumors, enrolling patients until an adequate number of MET-positive samples were available for analysis. The use of tissue from diagnosis, rather than from the time of recurrence, to assess MET status may also be considered a limitation.

In an exploratory biomarker analysis, HGF levels, MGMT promoter methylation status, and glioblastoma subtype were correlated with efficacy outcomes. High HGF levels by PCR or ISH seemed to be prognostic for worse OS and PFS, but potentially 

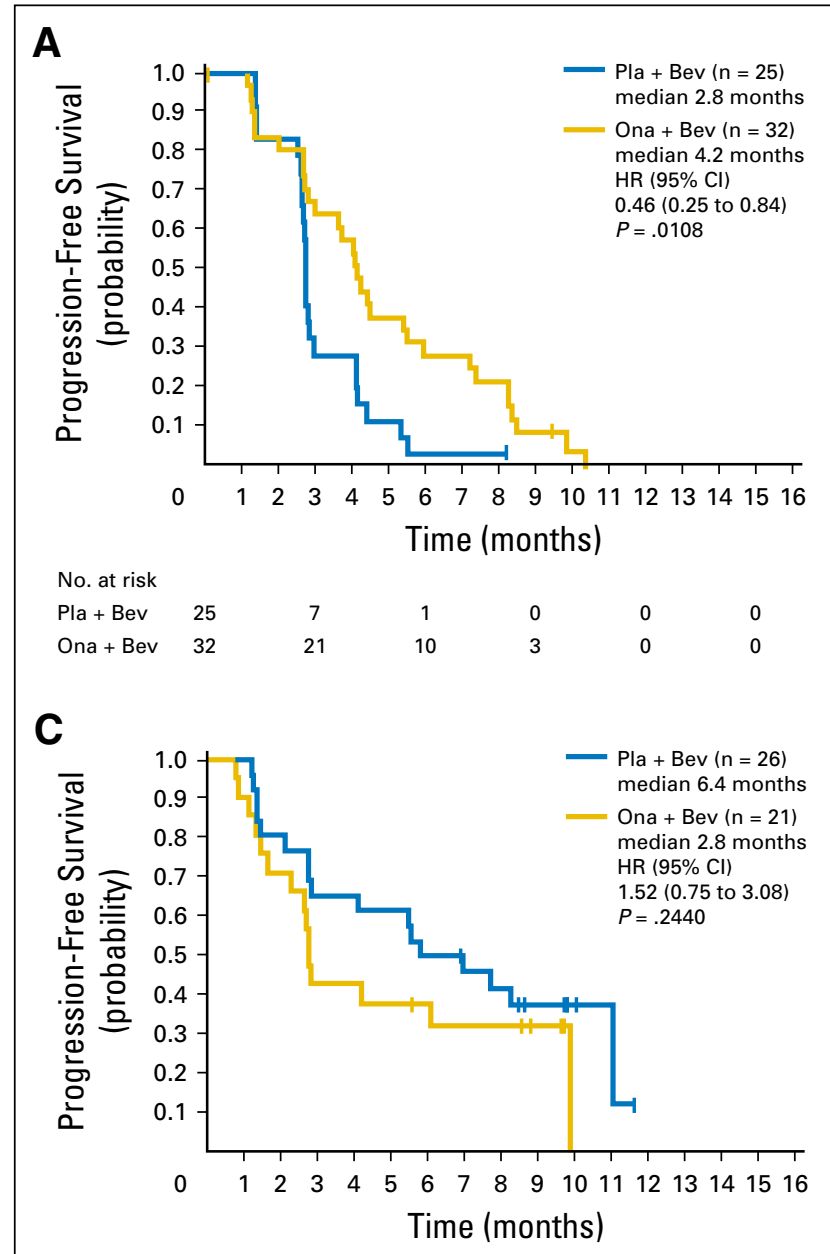

No. at risk

$\begin{array}{lcccccc}\text { Pla }+ \text { Bev } & 26 & 17 & 13 & 7 & 0 & 0 \\ \text { Ona }+ \text { Bev } & 21 & 9 & 7 & 3 & 0 & 0\end{array}$
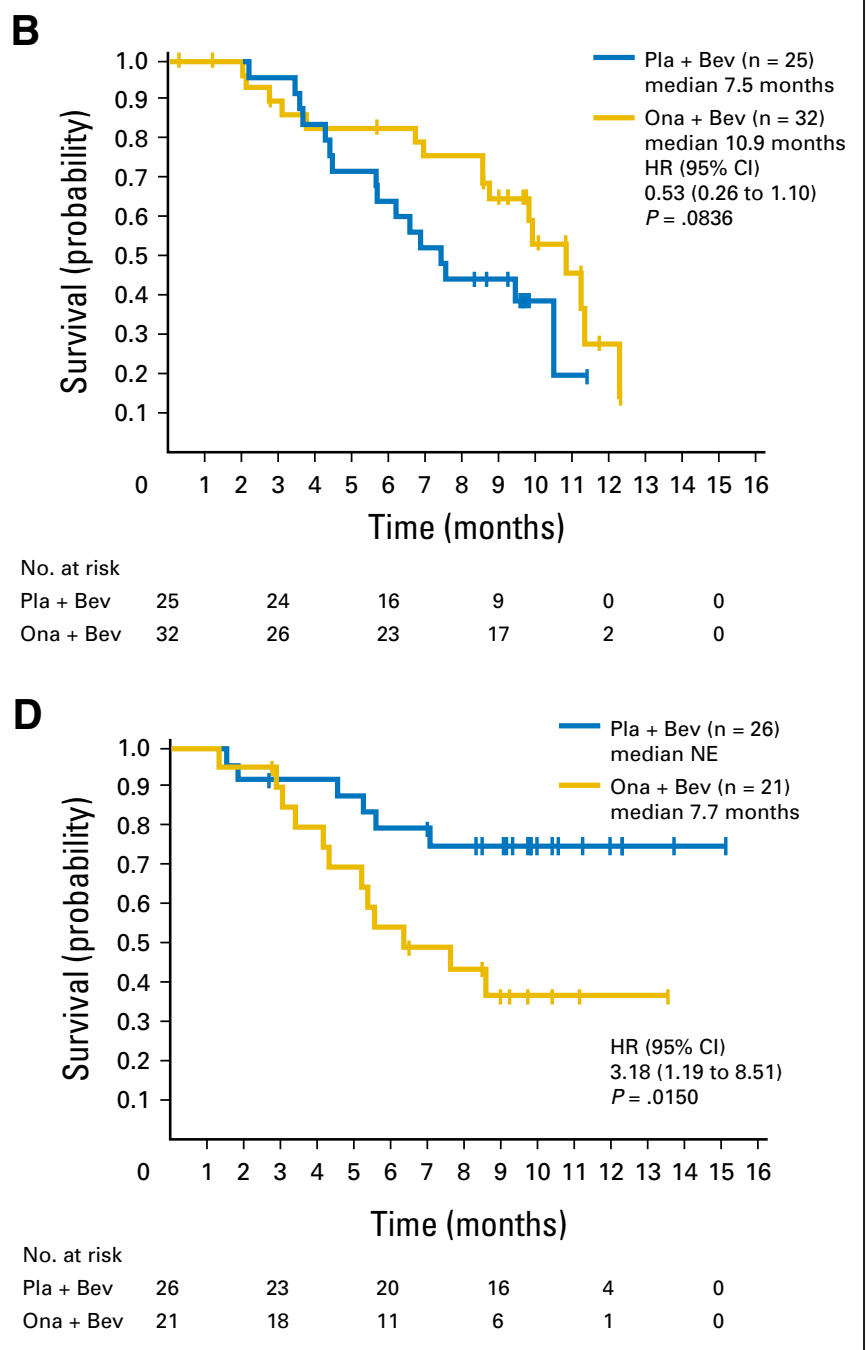

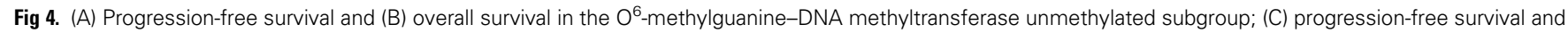

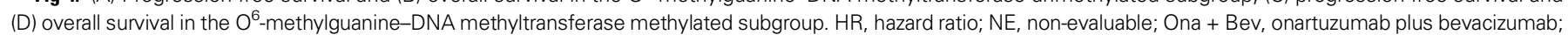
$\mathrm{Pla}+\mathrm{Bev}$, placebo plus bevacizumab.

predictive of superior efficacy with Ona + Bev compared with Pla + Bev. High HGF expression may indicate an aberrant MET pathway; in gastric cancer models, expression of HGF correlates with MET pathway activity and can predict efficacy of anti-MET agents. ${ }^{29}$ In hepatocellular carcinoma, circulating HGF levels correlate with decreased survival in untreated patients. ${ }^{30}$

Analysis of HGF in the phase III AVAglio study of radiotherapy, temozolomide plus bevacizumab or placebo in newly diagnosed gliomas showed that the mesenchymal subtype was associated with high HGF expression and reported that patients with the mesenchymal subtype had longer OS than those with other subtypes. ${ }^{16}$ In this onartuzumab study, HGF expression was associated with the mesenchymal subtype; however, not all mesenchymal tumors expressed high HGF levels and the mesenchymal phenotype was not associated with different outcomes compared with other phenotypes, most likely as a result of the bevacizumab backbone in both treatment arms.

Tumor-specific promoter methylation and silencing of MGMT enhances the efficacy of alkylating agents in tumor cells because the cells are unable to efficiently repair damage induced by these agents. ${ }^{26}$ The exploratory results in GO27819 suggest that the lack of MGMT methylation may be predictive for Ona + Bev outcomes (PFS and OS) in glioblastoma, although the OS results were not statistically significant. However, in the AVAglio study, no predictive influence of MGMT methylation status was observed on bevacizumab efficacy. ${ }^{14}$ MGMT silencing via methylation allows alkylating agents to cause cell death by crosslinking, thereby improving efficacy of these agents. ${ }^{26}$ In the absence of a direct biologic link between MGMT methylation and MET, other than a potentially additive effect on the apoptosis pathway, it is notable that the treatment effect with unmethylated MGMT was similar to the high HGF subgroup. Clearly, some potent prognostic factors influence the pathogenesis of glioblastoma, which need to be considered in future trial designs. Therefore, it is essential to appropriately size studies to enable evaluation of exploratory hypotheses.

Although the biomarker data presented above are compelling and should inform future trials targeting the MET pathway, their 


\begin{tabular}{|lcc|}
\hline \multicolumn{3}{|c|}{ Table 2. Safety Analysis } \\
\hline \multicolumn{1}{|c}{ AEs } & $\begin{array}{c}\text { Ona }+\mathrm{Bev} \\
(\mathrm{n}=65)^{*}\end{array}$ & $\begin{array}{c}\mathrm{Pla}+\mathrm{Bev} \\
(\mathrm{n}=64)^{*}\end{array}$ \\
\hline Grade $\geq 3$ AEs & $25(38.5)$ & $23(35.9)$ \\
Serious AEs & $20(30.8)$ & $19(29.7)$ \\
Nervous system disorders & $5(7.7)$ & $11(17.2)$ \\
Vascular disorders & $4(6.2)$ & $5(7.8)$ \\
Infections and infestations & $4(6.2)$ & $4(6.3)$ \\
GI disorders & $4(6.2)$ & $0(0.0)$ \\
Grade 5 AEs & $2(3.1)$ & $1(1.6)$ \\
AEs leading to withdrawal of any study drug & $7(10.8)$ & $4(6.3)$ \\
AEs leading to dose interruption of any study drug & $21(32.3)$ & $15(23.4)$ \\
\hline NOTE. All values are expressed as no. (\%). & \\
Abbreviations: AE, adverse event; Bev, bevacizumab; Ona, onartuzumab; Pla, \\
placebo. \\
*On the basis of treatment received if different than treatment randomly \\
assigned to receive.
\end{tabular}

exploratory nature and relatively small sample size make it difficult to infer firm conclusions on the clinical utility of $H G F$ expression or MGMT methylation as predictive markers in glioblastoma. Future prospective studies are necessary to validate these results.

In conclucion, in the GO27819 trial, there was no evidence of improved clinical benefit with the addition of onartuzumab to bevacizumab compared with bevacizumab plus placebo in ITT patients with recurrent glioblastoma. However, exploratory biomarker analyses suggested that patients with high $H G F$ expression or unmethylated MGMT may benefit from onartuzumab plus bevacizumab.

\section{AUTHORS' DISCLOSURES OF POTENTIAL CONFLICTS OF INTEREST}

Disclosures provided by the authors are available with this article at ascopubs.org/journal/jco.

\section{AUTHOR CONTRIBUTIONS}

Conception and design: Timothy Cloughesy, Carlos Bais, See-Chun Phan, David S. Shames

Provision of study materials or patients: Timothy Cloughesy, Gaetano Finocchiaro, Cristóbal Belda-Iniesta, Alba A. Brandes, Estela Pineda, Carmen Balana, David R. Macdonald, Kirsten Hopkins, Michael Weller Collection and assembly of data: Timothy Cloughesy, Gaetano Finocchiaro, Lawrence Recht, Alba A. Brandes, Estela Pineda, Tom Mikkelsen, Carmen Balana, David R. Macdonald, Kirsten Hopkins, Michael Weller, Carlos Bais, Jean-Marie Bruey, Hartmut Koeppen, Bo Liu, See-Chun Phan, David S. Shames

Data analysis and interpretation: Timothy Cloughesy, Cristóbal Belda-Iniesta, Alba A. Brandes, Olivier L. Chinot, Manfred Westphal, Michael Weller, Carlos Bais, Thomas Sandmann, Jean-Marie Bruey, Hartmut Koeppen, Bo Liu, Wendy Verret, See-Chun Phan,

David S. Shames

Manuscript writing: All authors

Final approval of manuscript: All authors

\section{REFERENCES}

1. Ostrom QT, Gittleman $H$, Liao $P$, et al: CBTRUS statistical report: Primary brain and central nervous system tumors diagnosed in the United States in 2007-2011. Neuro-oncol 16(Suppl 4):iv1-iv63, 2014

2. Walker C, Baborie A, Crooks D, et al: Biology, genetics and imaging of glial cell tumours. $\mathrm{Br} \mathrm{J}$ Radiol 84:S90-S106, 2011

3. Stupp R, Mason WP, van den Bent MJ, et al: Radiotherapy plus concomitant and adjuvant temozolomide for glioblastoma. N Engl J Med 352: 987-996, 2005

4. Weller M, van den Bent M, Hopkins $K$, et al: EANO guideline for the diagnosis and treatment of anaplastic gliomas and glioblastoma. Lancet Oncol 15:e395-e403, 2014

5. Verhaak RG, Hoadley KA, Purdom E, et al: Integrated genomic analysis identifies clinically relevant subtypes of glioblastoma characterized by abnormalities in PDGFRA, IDH1, EGFR, and NF1. Cancer Cell 17:98-110, 2010

6. Chaudhry IH, O'Donovan DG, Brenchley PEC, et al: Vascular endothelial growth factor expression correlates with tumour grade and vascularity in gliomas. Histopathology 39:409-415, 2001

7. Friedman HS, Prados MD, Wen PY, et al: Bevacizumab alone and in combination with irinotecan in recurrent glioblastoma. J Clin Oncol 27: 4733-4740, 2009

8. Kreisl TN, Kim L, Moore K, et al: Phase II trial of single-agent bevacizumab followed by bevacizumab plus irinotecan at tumor progression in recurrent glioblastoma. J Clin Oncol 27:740-745, 2009
9. Vredenburgh JJ, Desjardins A, Herndon JE II, et al: Bevacizumab plus irinotecan in recurrent glioblastoma multiforme. J Clin Oncol 25:4722-4729, 2007

10. Soffietti R, Trevisan E, Bertero L, et al: Bevacizumab and fotemustine for recurrent glioblastoma: A phase II study of AINO (Italian Association of Neuro-Oncology). J Neurooncol 116:533-541, 2014

11. Maree $K$, Simes J, Wheeler $H$, et al: A randomized phase II study of carboplatin and bevacizumab in recurrent glioblastoma multiforme (CABARET). Presented at the 2013 American Society of Clinical Oncology Annual Meeting, Chicago, IL, USA, May 31-June 4, 2013

12. Taal W, Oosterkamp HM, Walenkamp AM, et al: Single-agent bevacizumab or lomustine versus a combination of bevacizumab plus lomustine in patients with recurrent glioblastoma (BELOB trial): A randomised controlled phase 2 trial. Lancet Oncol 15: 943-953, 2014

13. Desjardins A, Reardon DA, Coan A, et al: Bevacizumab and daily temozolomide for recurrent glioblastoma. Cancer 118:1302-1312, 2012

14. Chinot OL, Wick W, Mason W, et al: Bevacizumab plus radiotherapy-temozolomide for newly diagnosed glioblastoma. N Engl J Med 370:709-722, 2014

15. Gilbert MR, Dignam JJ, Armstrong TS, et al: A randomized trial of bevacizumab for newly diagnosed glioblastoma. N Engl J Med 370:699-708, 2014

16. Sandmann T, Bourgon R, Garcia J, et al: Patients with proneural glioblastoma may derive overall survival benefit from the addition of bevacizumab to first-line radiotherapy and temozolomide: Retrospective analysis of the AVAglio trial. J Clin Oncol 33:2735-44, 2015

17. Blumenschein GR Jr, Mills GB, GonzalezAngulo AM: Targeting the hepatocyte growth factorcMET axis in cancer therapy. J Clin Oncol 30:3287-3296, 2012

18. Lu KV, Bergers G: Mechanisms of evasive resistance to anti-VEGF therapy in glioblastoma. CNS Oncol 2:49-65, 2013

19. Kulig K, McLendon RE, Locke SC, et al: MET and ALK in glioblastoma multiforme (GBM): Comparison of IHC and FISH. J Clin Oncol 30, 2012 (suppl; abstr 2021)

20. Koochekpour S, Jeffers M, Rulong S, et al: Met and hepatocyte growth factor/scatter factor expression in human gliomas. Cancer Res 57: 5391-5398, 1997

21. Xie $Q$, Bradley $R$, Kang $L$, et al: Hepatocyte growth factor (HGF) autocrine activation predicts sensitivity to MET inhibition in glioblastoma. Proc Natl Acad Sci USA 109:570-575, 2012

22. Garnett J, Chumbalkar V, Vaillant B, et al: Regulation of HGF expression by $\triangle$ EGFR-mediated c-Met activation in glioblastoma cells. Neoplasia 15: 73-84, 2013

23. Martens T, Schmidt NO, Eckerich $C$, et al: $A$ novel one-armed anti-c-Met antibody inhibits glioblastoma growth in vivo. Clin Cancer Res 12:6144-6152, 2006

24. Wen PY, Macdonald DR, Reardon DA, et al: Updated response assessment criteria for high-grade gliomas: Response Assessment in Neuro-Oncology Working Group. J Clin Oncol 28:1963-1972, 2010

25. Koeppen $\mathrm{H}, \mathrm{Yu}$ W, Zha J, et al: Biomarker analyses from a placebo-controlled phase II study evaluating erlotinib \pm onartuzumab in advanced non-small 
cell lung cancer: MET expression levels are predictive of patient benefit. Clin Cancer Res 20:4488-4498, 2014

26. Weller M, Stupp R, Reifenberger G, et al: MGMT promoter methylation in malignant gliomas: Ready for personalized medicine? Nat Rev Neurol 6:39-51, 2010

27. Cankovic M, Mikkelsen $T$, Rosenblum ML, et al: A simplified laboratory validated assay for
MGMT promoter hypermethylation analysis of glioma specimens from formalin-fixed paraffin-embedded tissue. Lab Invest 87:392-397, 2007

28. Weller $M$, Cloughesy $T$, Perry JR, et al: Standards of care for treatment of recurrent glioblastoma-Are we there yet? Neuro-oncol 15:4-27, 2013
29. Hack SP, Bruey JM, Koeppen H: HGF/METdirected therapeutics in gastroesophageal cancer: $A$ review of clinical and biomarker development. Oncotarget 5:2866-2880, 2014

30. Goyal L, Muzumdar MD, Zhu AX: Targeting the HGF/c-MET pathway in hepatocellular carcinoma. Clin Cancer Res 19:2310-2318, 2013

\section{Affiliations}

Timothy Cloughesy, University of California, Los Angeles; Lawrence Recht, Stanford Cancer Center, Stanford; Carlos Bais, Thomas Sandmann, Jean-Marie Bruey, Hartmut Koeppen, Bo Liu, Wendy Verret, See-Chun Phan, and David S. Shames, Genentech, South San Francisco, CA; Tom Mikkelsen, Henry Ford Hospital, Detroit, MI; Gaetano Finocchiaro, Istituto Neurologico Carlo Besta, Milan; Alba A. Brandes, Azienda Unità Sanitaria Locale-Istituto di Ricovero e Cura a Carattere Scientifico Institute of Neurologic Sciences, Bologna, Italy; Cristóbal Belda-Iniesta, Centro Integral Oncológico Clara Campal, University Hospital HM Sanchinarro, Madrid; Estela Pineda, Hospital Clinic of Barcelona; Carmen Balana, Institut Català d'Oncologia Badalona Hospital Germans Trias I Pujol, Barcelona, Spain; Olivier L. Chinot, Aix-Marseille Université Assistance Publique-Hôpitaux de Marseille, Marseille, France; David R. Macdonald, London Health Sciences Centre, London, Ontario, Canada; Manfred Westphal, University Clinic Hamburg-Eppendorf, Hamburg, Germany; Kirsten Hopkins, Bristol Haematology and Oncology Centre, Bristol, United Kingdom; and Michael Weller, University Hospital Zurich, Zurich, Switzerland.

Funded by F. Hoffmann-La Roche.

\section{Support}

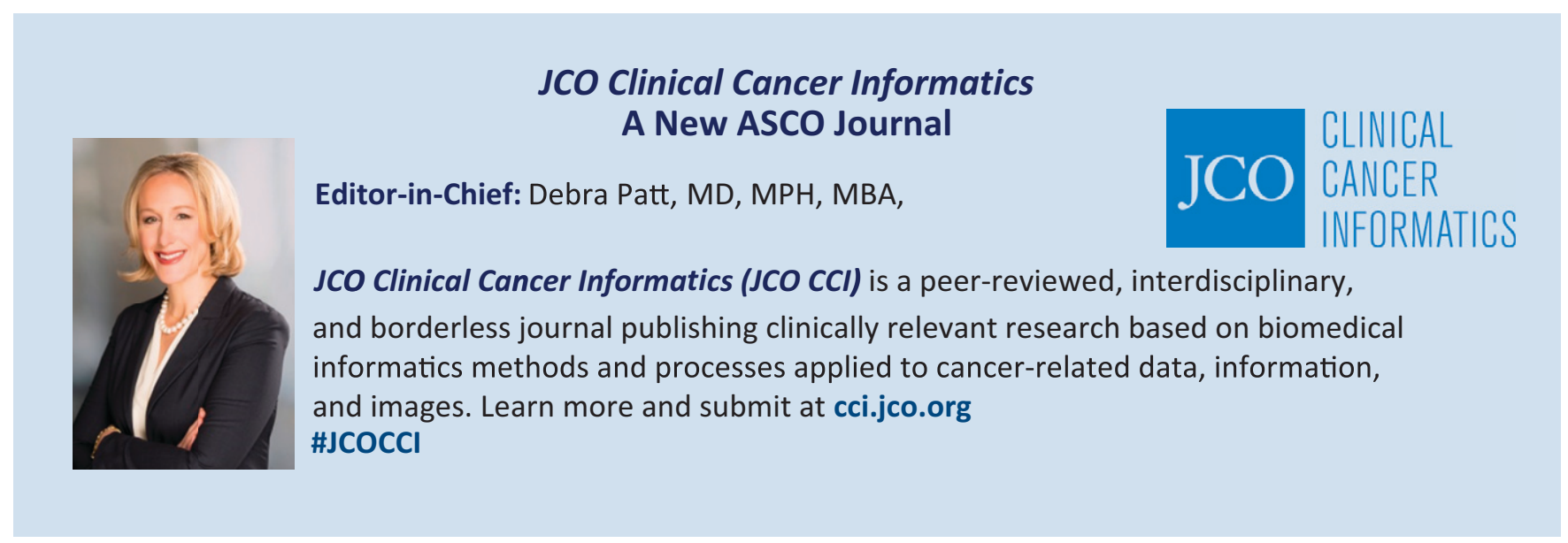




\section{AUTHORS' DISCLOSURES OF POTENTIAL CONFLICTS OF INTEREST}

Randomized, Double-Blind, Placebo-Controlled, Multicenter Phase II Study of Onartuzumab Plus Bevacizumab Versus Placebo Plus Bevacizumab in Patients With Recurrent Glioblastoma: Efficacy, Safety, and Hepatocyte Growth Factor and $\mathrm{O}^{6}$-Methylguanine-DNA Methyltransferase Biomarker Analyses

The following represents disclosure information provided by authors of this manuscript. All relationships are considered compensated. Relationships are self-held unless noted. I = Immediate Family Member, Inst = My Institution. Relationships may not relate to the subject matter of this manuscript. For more information about ASCO's conflict of interest policy, please refer to www.asco.org/rwc or ascopubs.org/jco/site/ifc.

Timothy Cloughesy

Consulting or Advisory Role: Genentech, Celgene, Tocagen, VBL Therapeutics, NewGen Therapeutics, Novartis, Upsher-Smith, Proximagen, Lpath, StemCycle, Amgen, INSYS Therapeutics

Expert Testimony: Roche

Gaetano Finocchiaro

Travel, Accommodations, Expenses: Roche for 19th Annual Scientific Meeting and Education Day of the Society for Neuro-Oncology (2014).

Cristóbal Belda-Iniesta

Employment: HM Hospitales, Merck Serono

Honoraria: Roche, AbbVie, Merck Serono

Consulting or Advisory Role: Roche, AbbVie

Lawrence Recht

Consulting or Advisory Role: Nektar Therapeutics

Research Funding: Nektar Therapeutics, Berg LLC

Alba A. Brandes

Travel, Accommodations, Expenses: Merck Serono, Pfizer, Roche, AIOM

Estela Pineda

No relationship to disclose

Tom Mikkelsen

Honoraria: Genentech

Consulting or Advisory Role: Genentech

Travel, Accommodations, Expenses: Genentech

Olivier L. Chinot

Honoraria: AbbVie, Celldex, Ipsen, Novartis

Consulting or Advisory Role: Roche, Ipsen

Research Funding: Roche

Patents, Royalties, Other Intellectual Property: Aix Marseille University

Travel, Accommodations, Expenses: Roche, SERVIER

\section{Carmen Balana}

Consulting or Advisory Role: Ferrer, Celldex

David R. Macdonald

Honoraria: Roche Canada

Consulting or Advisory Role: Roche Canada, VBL Therapeutics

Manfred Westphal

Consulting or Advisory Role: Novocure, Bristol-Myers Squibb

Travel, Accommodations, Expenses: Bristol-Myers Squibb

Kirsten Hopkins

No relationship to disclose

\section{Michael Weller}

Honoraria: Merck Serono, Roche, Eli Lilly, MSD, ImmunoCellular Therapeutics

Consulting or Advisory Role: Roche, Merck Serono, MagForce, Isarna Therapeutics, Celldex, Eli Lilly, Northwest Biotherapeutics, Pfizer, Teva Research Funding: Bayer (Inst), Isarna Therapeutics (Inst), Roche (Inst), Merck Serono (Inst), Piqur (Inst), Actelion (Inst), Acceleron Pharma (Inst), Novocure (Inst)

\section{Carlos Bais}

Employment: Genentech, Medimmune/AstraZeneca

Stock or Other Ownership: Genentech, AstraZeneca

Honoraria: Genentech, Medimmune/AstraZeneca

Patents, Royalties, Other Intellectual Property: Genentech

Thomas Sandmann

Employment: Genentech, Verily Life Science

Stock or Other Ownership: Genentech

Patents, Royalties, Other Intellectual Property: Coinventor on several patent applications that have been filed as part of employment with Genentech

Jean-Marie Bruey

Employment: Genentech

Stock or Other Ownership: Roche Holdings

Hartmut Koeppen

Employment: Roche

Stock or Other Ownership: Roche

Patents, Royalties, Other Intellectual Property: Roche

Bo Liu

Employment: Roche

Wendy Verret

Employment: Genentech

See-Chun Phan

Employment: Genentech

Stock or Other Ownership: Roche Holdings

Patents, Royalties, Other Intellectual Property: Genentech

Travel, Accommodations, Expenses: Genentech

David S. Shames

Employment: Genentech

Stock or Other Ownership: Roche Holdings

Patents, Royalties, Other Intellectual Property: UT Southwestern, Alnylam, Genentech 


\section{Acknowledgment}

Support for third-party writing assistance for this manuscript was provided by F. Hoffmann-La Roche Ltd.

\section{Appendix}

\section{Gene Expression Analysis on the NanoString Platform}

Transcript levels in formalin-fixed paraffin-embedded material were assayed on the NanoString gene expression platform (NanoString Technologies, Seattle, WA), as described previously. ${ }^{16}$ Data preprocessing was performed using the NanoStringQCPro Bioconductor package (version 1.0.1; http://www.bioconductor.org/packages/release/bioc/html/NanoStringQCPro.html) and the R programming language (version 3.1; http://www.r-project.org). In brief, for each sample, unspecific background was estimated as the mean expression across the negative-control probes and subtracted from the raw counts. Background-corrected counts were $\log ^{2}$ transformed and centered on the median expression of all probes designed to target endogenous transcripts (global median normalization).

\section{Gene Expression Subtype Classification}

Subtype classification was performed by assigning each sample to the reference centroid with the highest Pearson correlation across the normalized expression of 31 classifier genes, ${ }^{16}$ as described previously (Phillips HS, et al: Cancer Cell 9:157-173, 2006). Samples without positive correlation to any reference centroid remained unclassified.

\section{Differential Hepatocyte Growth Factor (HGF) Expression}

Differential expression of HGF between glioblastoma subtypes was assessed by comparing normalized gene expression scores on the $\log ^{2}$ scale. Ninety-five percent CIs for mean HGF expression in each subgroup were estimated on the basis of the Student $t$ test distribution. $P$ values were obtained by contrasting $H G F$ expression in mesenchymal samples with those in each of the other subtypes using a two-sided $t$ test.

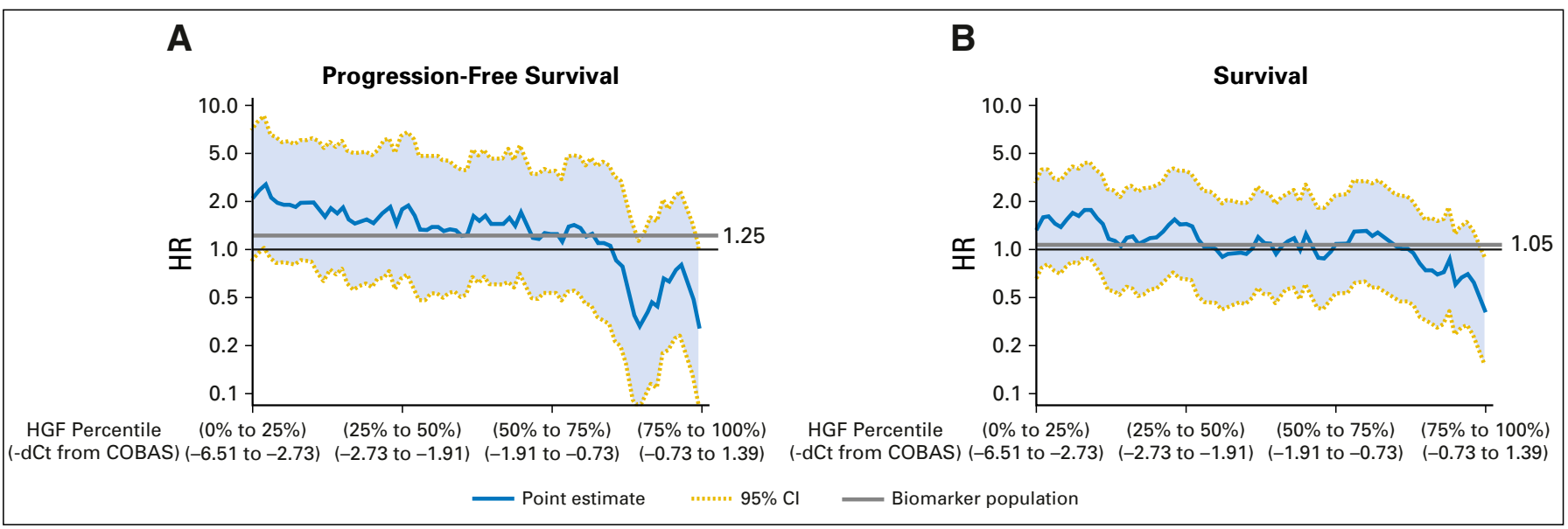

Fig A1. Subpopulation treatment effect pattern plots for hepatocyte growth factor (HGF). dCT, delta cycle threshold; HR, hazard ratio. 


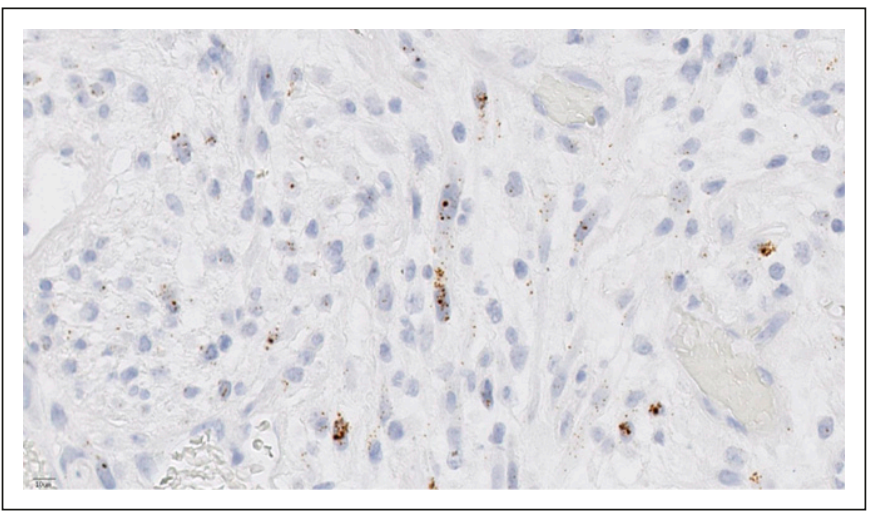

Fig A2. Representative image of hepatocyte growth factor in situ hybridization staining.
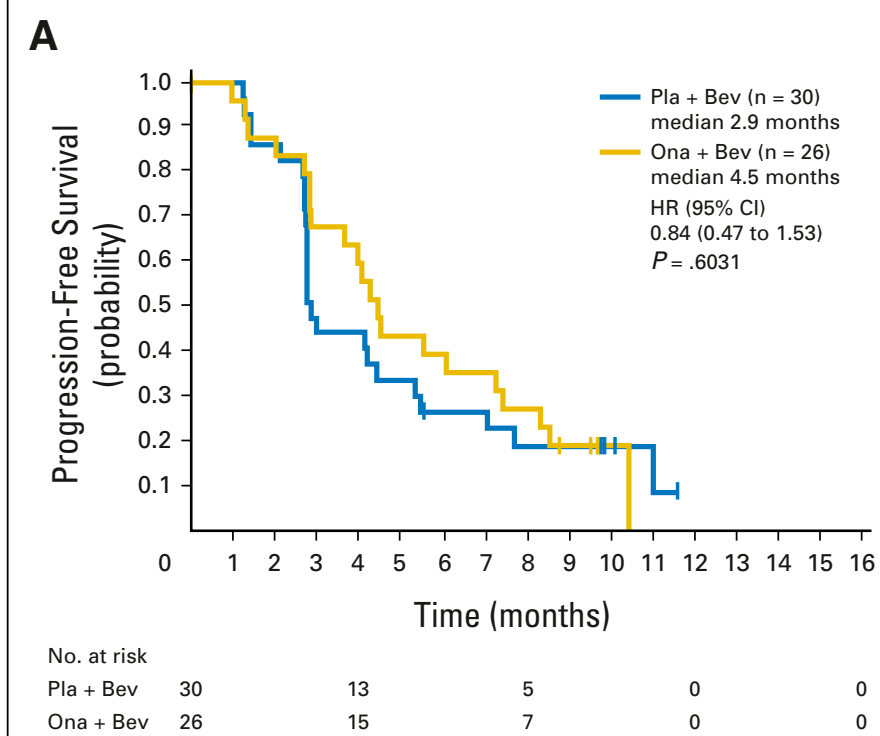

C

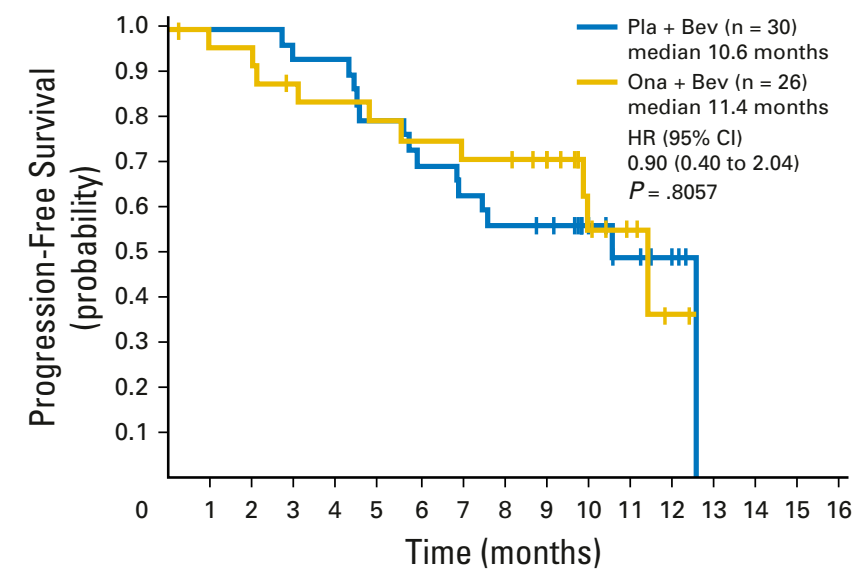

No. at risk

$\begin{array}{llllll}\mathrm{Pla}+\mathrm{Bev} & 30 & 28 & 17 & 3 & 0 \\ \text { Ona }+ \text { Bev } & 26 & 20 & 17 & 1 & 0\end{array}$
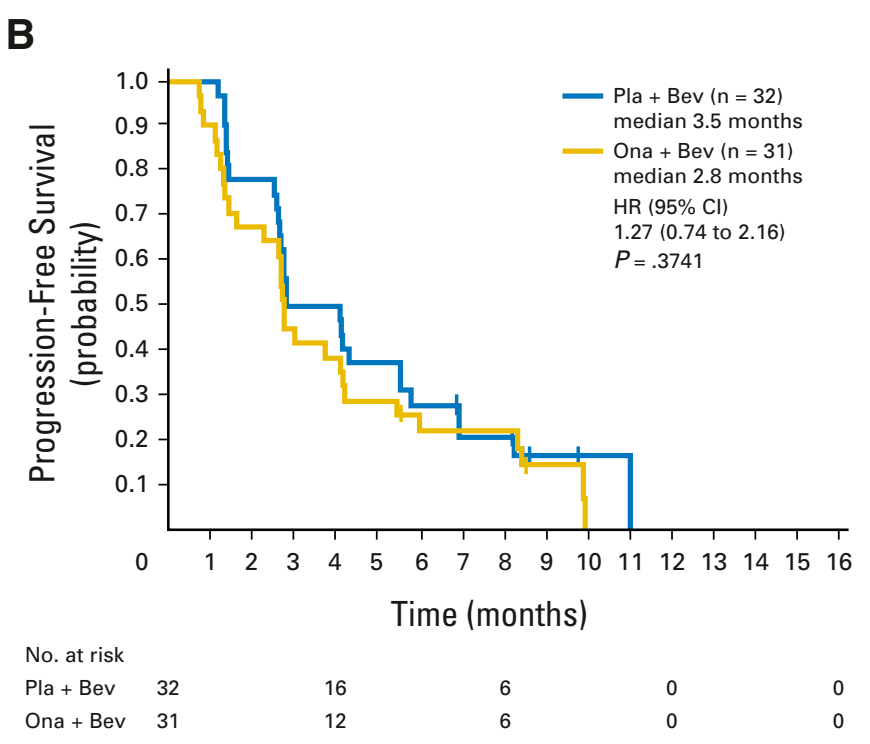

\section{B}

D

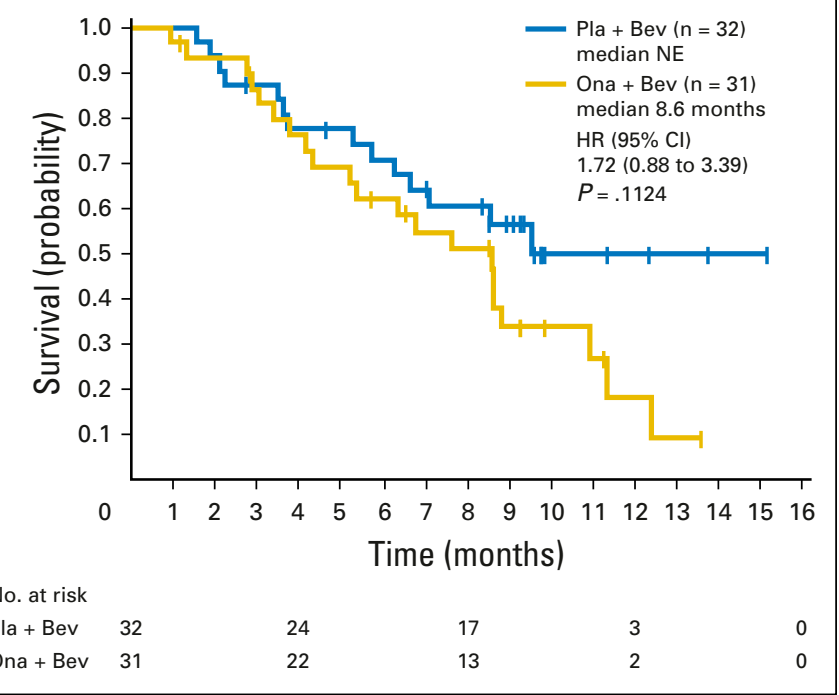

Fig A3. (A) Progression-free survival in mesenchymal subgroup and (B) nonmesenchymal subgroup and (C) overall survival in mesenchymal subgroup and (D) nonmesenchymal subgroup. HR, hazard ratio; NE, non-evaluable; Ona + Bev, onartuzumab plus bevacizumab; Pla + Bev, placebo plus bevacizumab. 


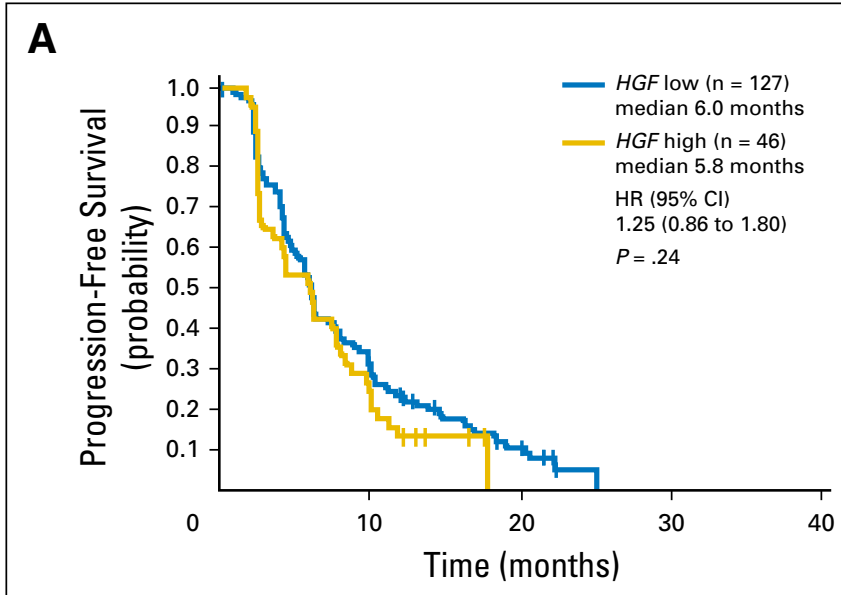

No. at risk $\begin{array}{lccccc}\text { HGF low } & 127 & 38 & 9 & 0 & 0 \\ \text { HGF high } & 46 & 11 & 0 & 0 & 0\end{array}$

C

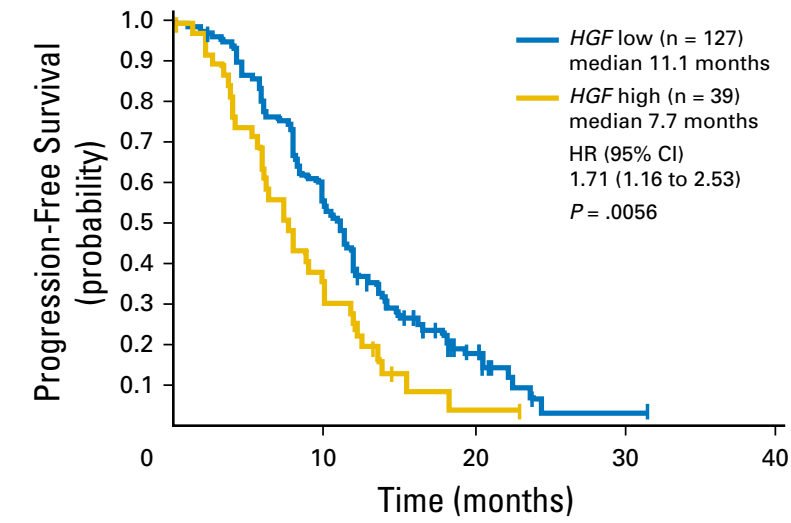

No. at risk

$\begin{array}{lccccc}\text { HGF low } & 127 & 70 & 14 & 2 & 0\end{array}$

B

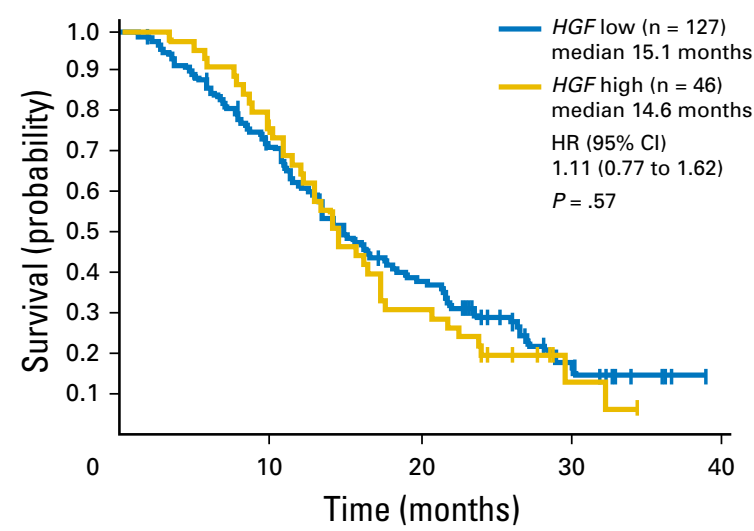

$\begin{array}{lccccc}\text { No. at risk } & & & & & \\ \text { HGF low } & 127 & 90 & 47 & 14 & 0 \\ \text { HGF high } & 46 & 36 & 15 & 3 & 0\end{array}$

D

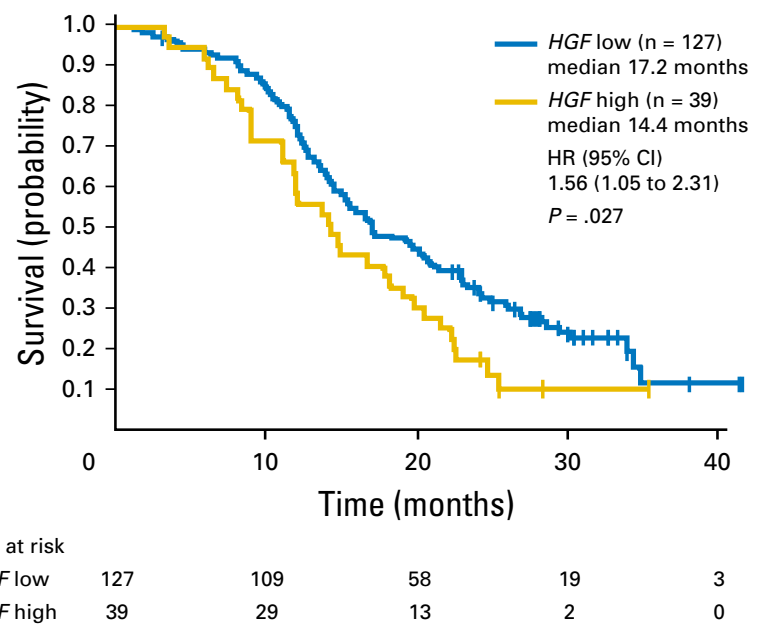

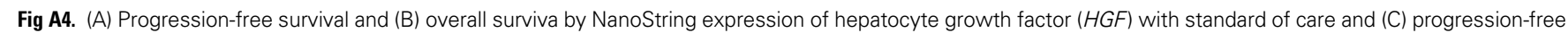
survival and (D) overall survival with standard of care plus bevacizumab in the Avastin in Glioblastoma (AVAglio) study. HR, hazard ratio. 


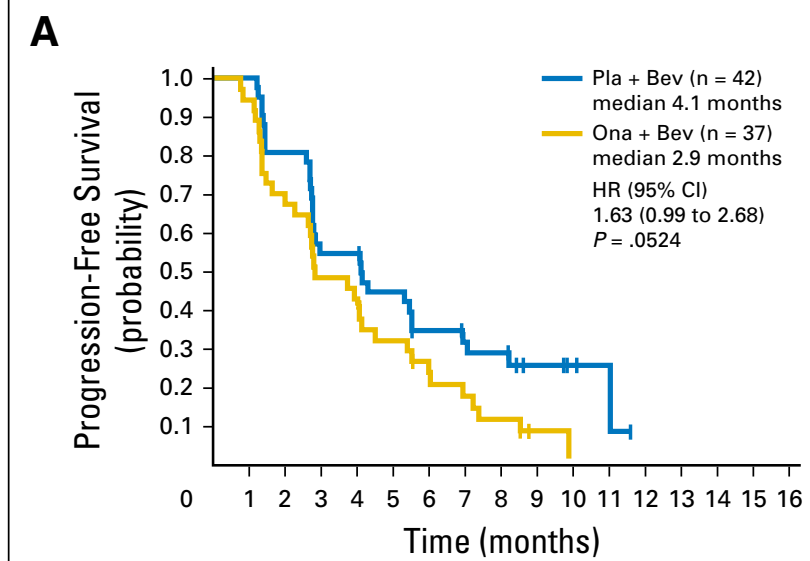

No. at risk

$\begin{array}{llcccc}\text { Pla }+ \text { Bev } & 42 & 23 & 10 & 0 & 0 \\ \text { Ona }+ \text { Bev } & 37 & 16 & 4 & 0 & 0\end{array}$

C

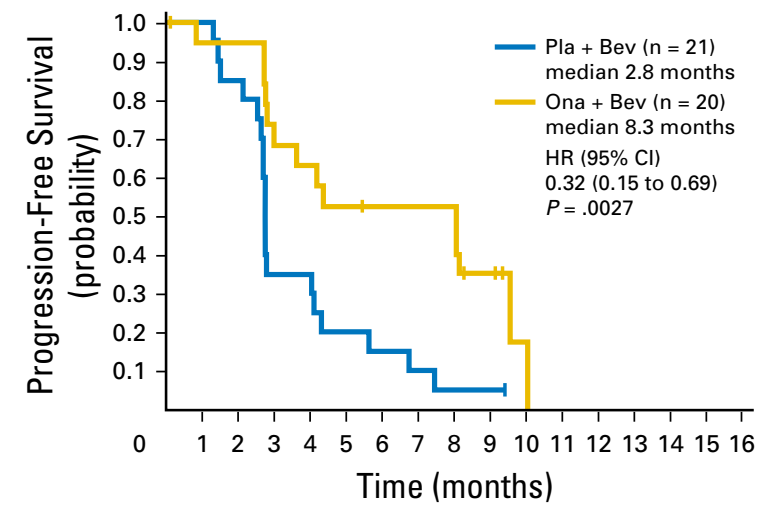

No. at risk

$\begin{array}{lccccc}\text { Pla }+ \text { Bev } & 21 & 7 & 1 & 0 & 0 \\ \text { Ona }+ \text { Bev } & 20 & 12 & 9 & 0 & 0\end{array}$

B

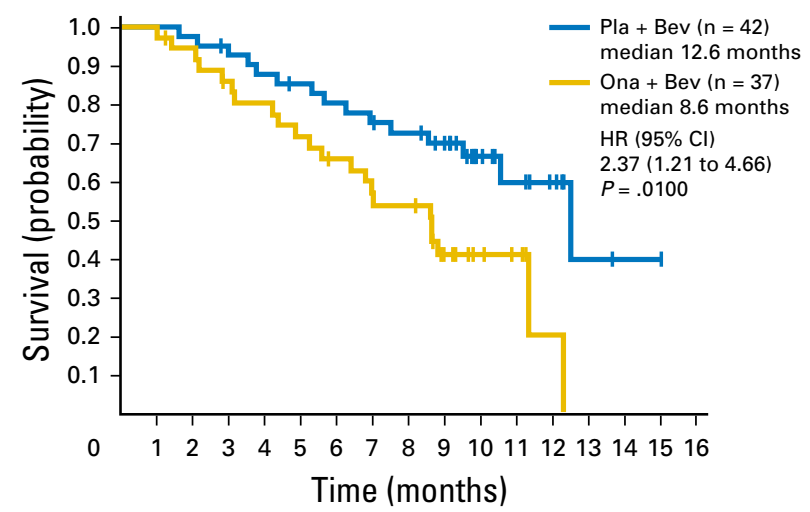

No. at risk

$\begin{array}{llllll}\text { Pla }+ \text { Bev } & 42 & 36 & 26 & 6 & 0 \\ \text { Ona }+ \text { Bev } & 37 & 26 & 18 & 1 & 0\end{array}$

Fig A5. (A) Progression-free survival and (B) overall survival in the low hepatocyte growth factor by in situ hybridization subgroup; (C) progression-free survival and

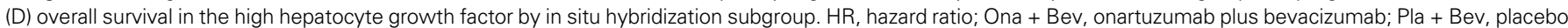
plus bevacizumab. 


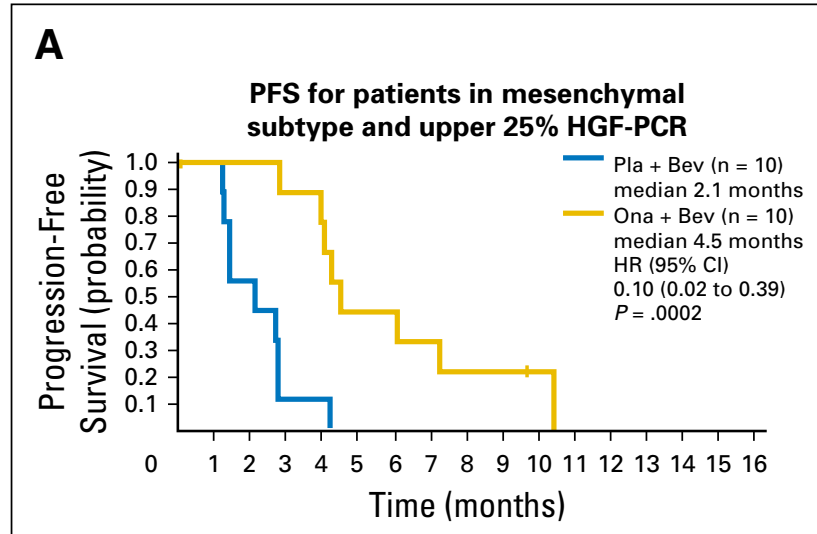

No. at risk

$\begin{array}{llllll}\text { Pla }+ \text { Bev } & 10 & 1 & 0 & 0 & 0 \\ \text { Ona }+ \text { Bev } & 10 & 7 & 2 & 0 & 0\end{array}$

C

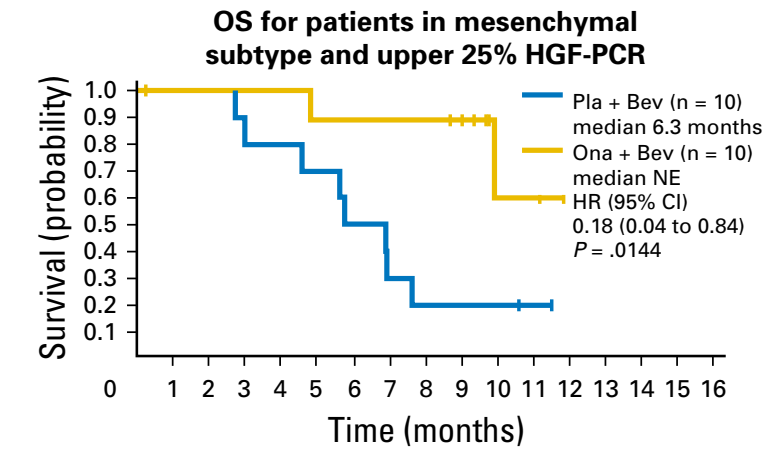

No. at risk

$\begin{array}{llllll}\text { Pla }+ \text { Bev } & 10 & 8 & 2 & 0 & 0 \\ \text { Ona }+ \text { Bev } & 10 & 9 & 8 & 0 & 0\end{array}$

E

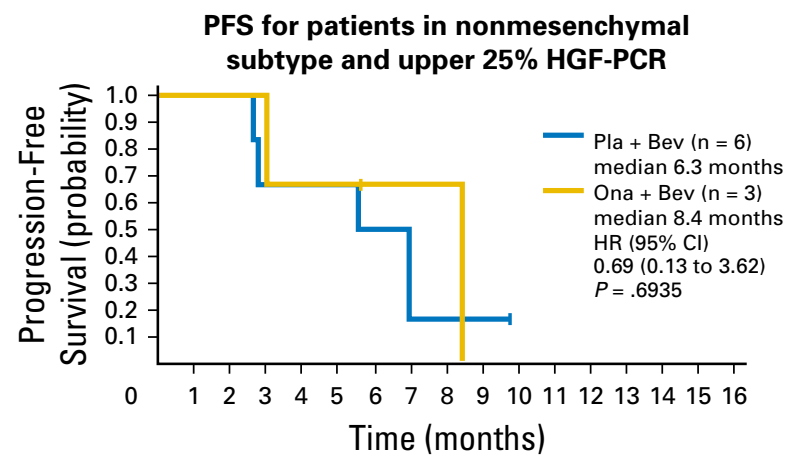

No. at risk

$\begin{array}{llllll}\text { Pla }+ \text { Bev } & 6 & 4 & 1 & 0 & 0 \\ \text { Ona + Bev } & 3 & 2 & 1 & 0 & 0\end{array}$

B

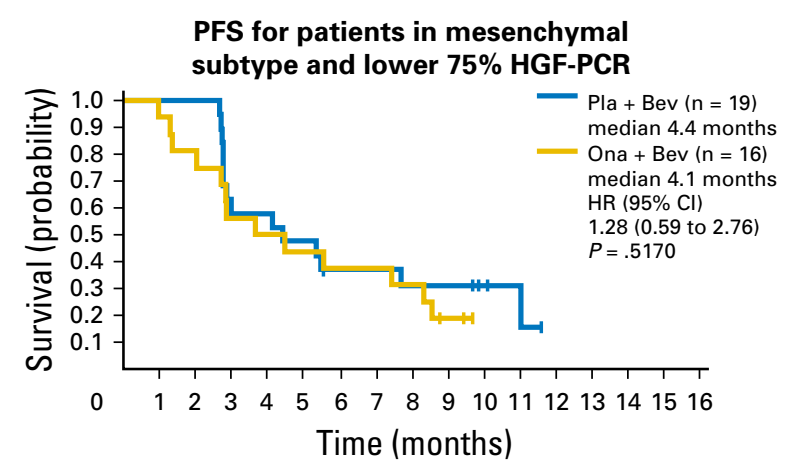

No. at risk

$\begin{array}{lccccc}\text { Pla }+ \text { Bev } & 19 & 11 & 5 & 0 & 0 \\ \text { Ona }+ \text { Bev } & 16 & 8 & 5 & 0 & 0\end{array}$

D

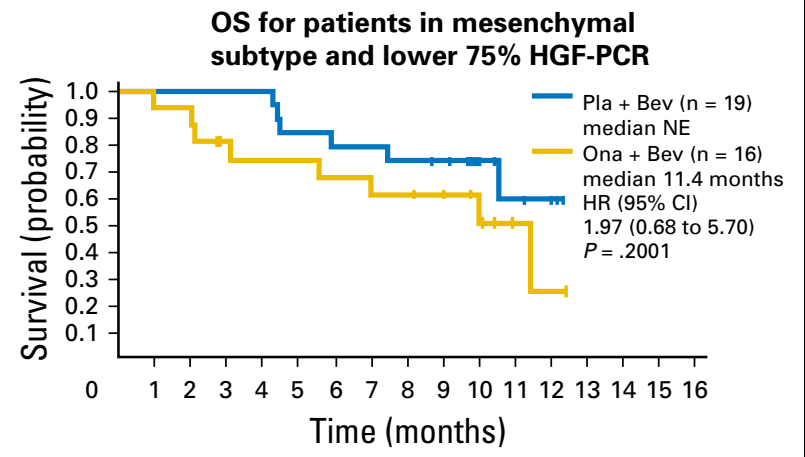

No. at risk

$\begin{array}{llllll}\text { Pla }+ \text { Bev } & 19 & 19 & 14 & 2 & 0 \\ \text { Ona + Bev } & 16 & 11 & 9 & 1 & 0\end{array}$

$\mathbf{F}$

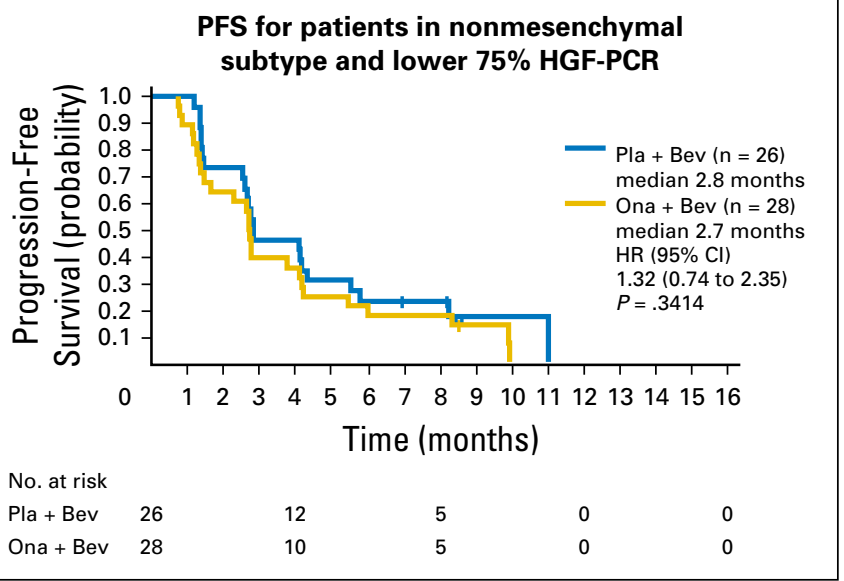

Fig A6. (A to D) Progression-free survival (PFS) and overall survival (OS) in mesenchymal and (E to $\mathrm{H}$ ) nonmesenchymal subgroups stratified by hepatocyte growth factor

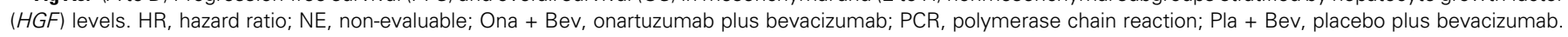




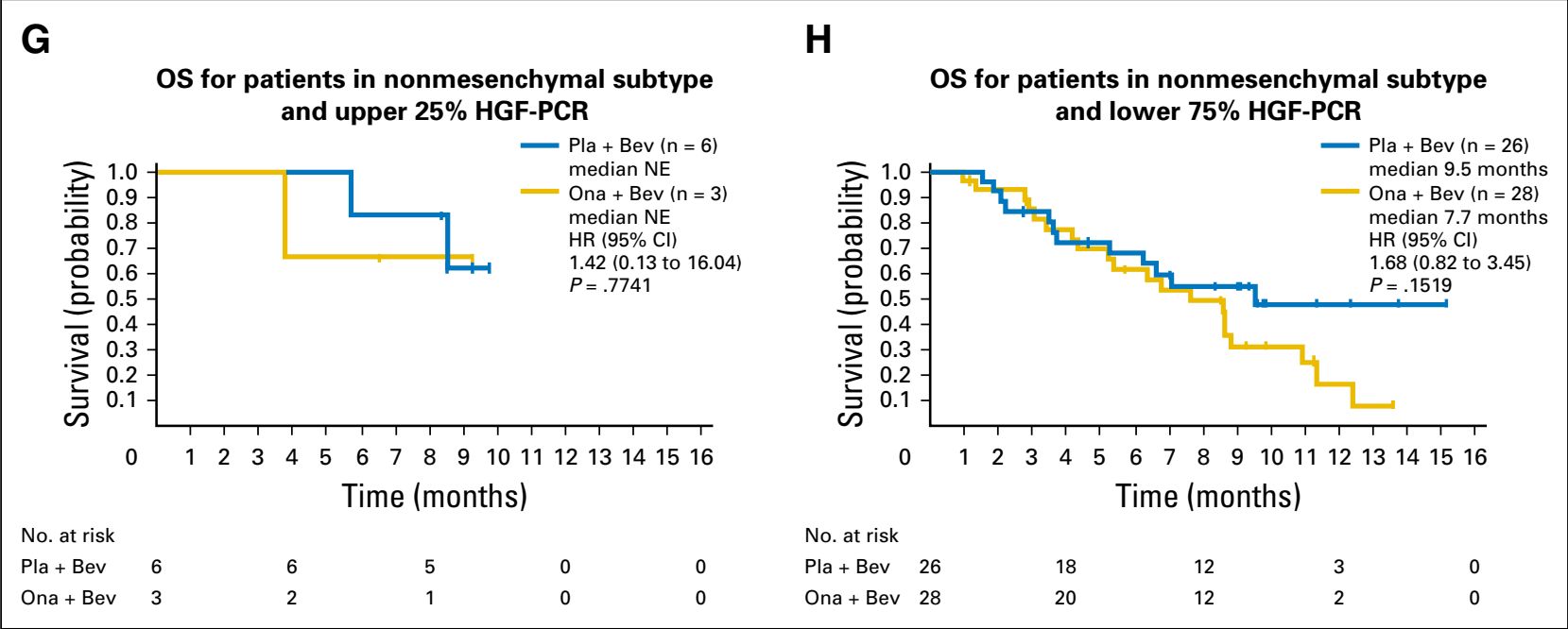

Fig A6. (Continued).
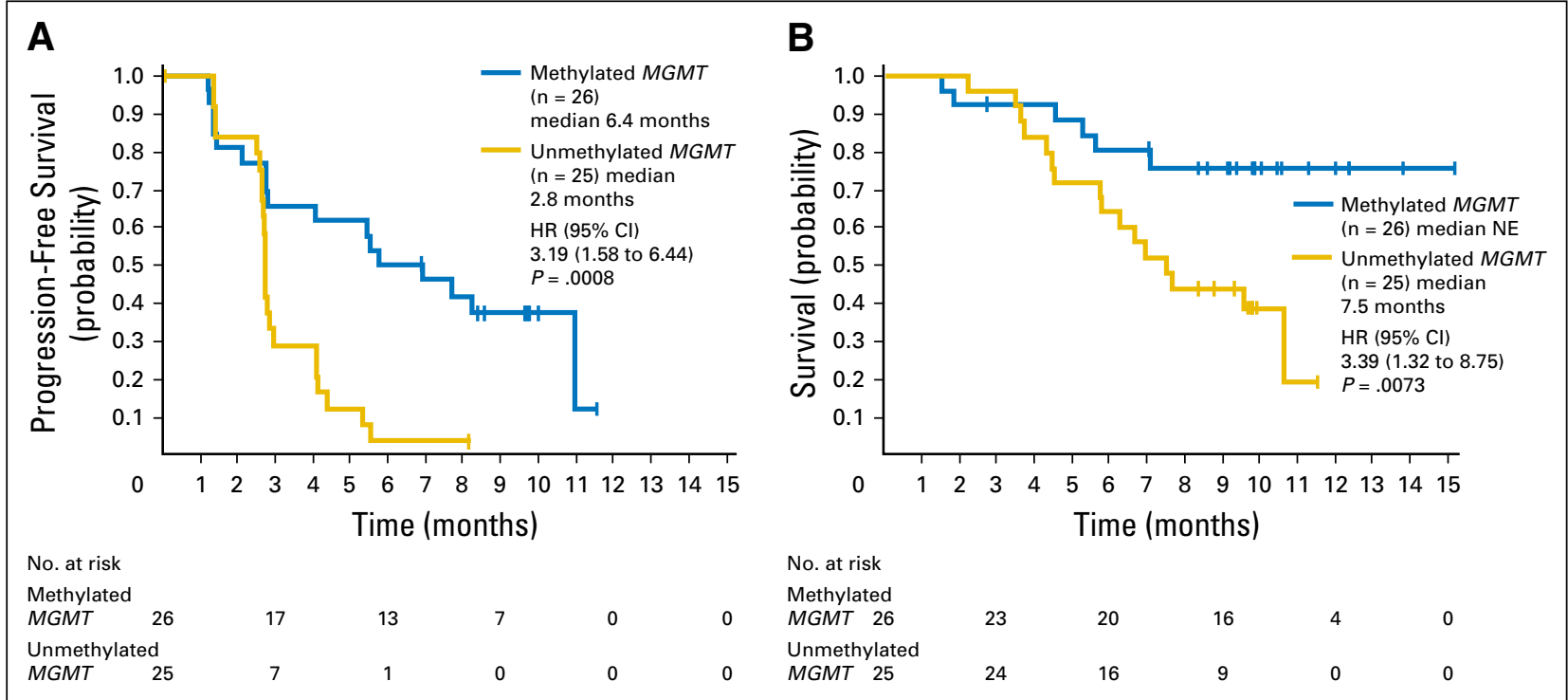

Fig A7. (A) Progression-free survival and (B) overall survival by $\mathrm{O}^{6}$-methylguanine-DNA methyltransferase (MGMT) methylation status in the placebo plus bevacizumab arm. HR, hazard ratio; NE, non-evaluable. 


\begin{tabular}{|c|c|c|c|c|c|}
\hline Subtype & No. & $\begin{array}{l}\% \text { Frequency in Sample } \\
\text { Population }(n=119)\end{array}$ & LSMEAN & $95 \% \mathrm{Cl}$ & $P$ (ref: mesenchymal) \\
\hline Mesenchymal & 56 & 47.1 & 0.620710 & 0.4997 to 0.7417 & \\
\hline Proliferative & 20 & 16.8 & 0.198178 & -0.0025 to 0.3989 & $<.001$ \\
\hline Proneural & 28 & 23.5 & 0.336095 & 0.1665 to 0.5057 & .0079 \\
\hline Unclassified & 15 & 12.6 & 0.377946 & 0.1462 to 0.6097 & .0684 \\
\hline
\end{tabular}

\begin{tabular}{|c|c|c|c|c|c|}
\hline Subtype & No. & $\begin{array}{c}\text { \% Frequency in Sample } \\
\text { Population }(n=119)\end{array}$ & LSMEAN & $95 \% \mathrm{Cl}$ & $P$ (ref: mesenchymal) \\
\hline Mesenchymal & 56 & 47.1 & 6.7279 & 6.3964 to 7.0593 & \\
\hline Proliferative & 20 & 16.8 & 5.3263 & 4.8257 to 5.8269 & $<.001$ \\
\hline Proneural & 28 & 23.5 & 5.9504 & 5.4167 to 6.4841 & .0149 \\
\hline Unclassified & 15 & 12.6 & 6.4224 & 5.9212 to 6.9235 & .2946 \\
\hline
\end{tabular}

\begin{tabular}{|c|c|c|c|c|c|}
\hline Subtype & No. & $\begin{array}{l}\% \text { Frequency in Sample } \\
\text { Population }(n=339)\end{array}$ & LSMEAN & $95 \% \mathrm{Cl}$ & $P$ (ref: mesenchymal) \\
\hline Mesenchymal & 139 & 41.0 & 6.3524 & 6.0206 to 6.6842 & \\
\hline Proliferative & 58 & 17.1 & 5.4113 & 4.8282 to 5.9944 & .0062 \\
\hline Proneural & 103 & 30.3 & 5.6896 & 5.2795 to 6.0997 & .0136 \\
\hline Unclassified & 39 & 11.5 & 6.7067 & 6.1210 to 7.2924 & .2933 \\
\hline
\end{tabular}

Table A4. High and Low Expression of Hepatocyte Growth Factor as Assessed by Polymerase Chain Reaction or In Situ Hybridization

\begin{tabular}{lccc}
\hline Expression Level & HGF by PCR, Upper 25\% & HGF by PCR, Lower 75\% & Total \\
\hline HGF by ISH, 2+/3+ & $19(63.3)$ & $22(25.3)$ & $41(35.0)$ \\
HGF by ISH, 0/1+ & $11(36.7)$ & $65(74.7)$ & $76(65.0)$ \\
Total & $30(25.6)$ & $87(74.4)$ & 117 \\
\hline
\end{tabular}

NOTE. All values are expressed as no. (\%).

Abbreviations: HGF, hepatocyte growth factor; ISH, in situ hybridization; PCR, polymerase chain reaction. 
Cloughesy et al

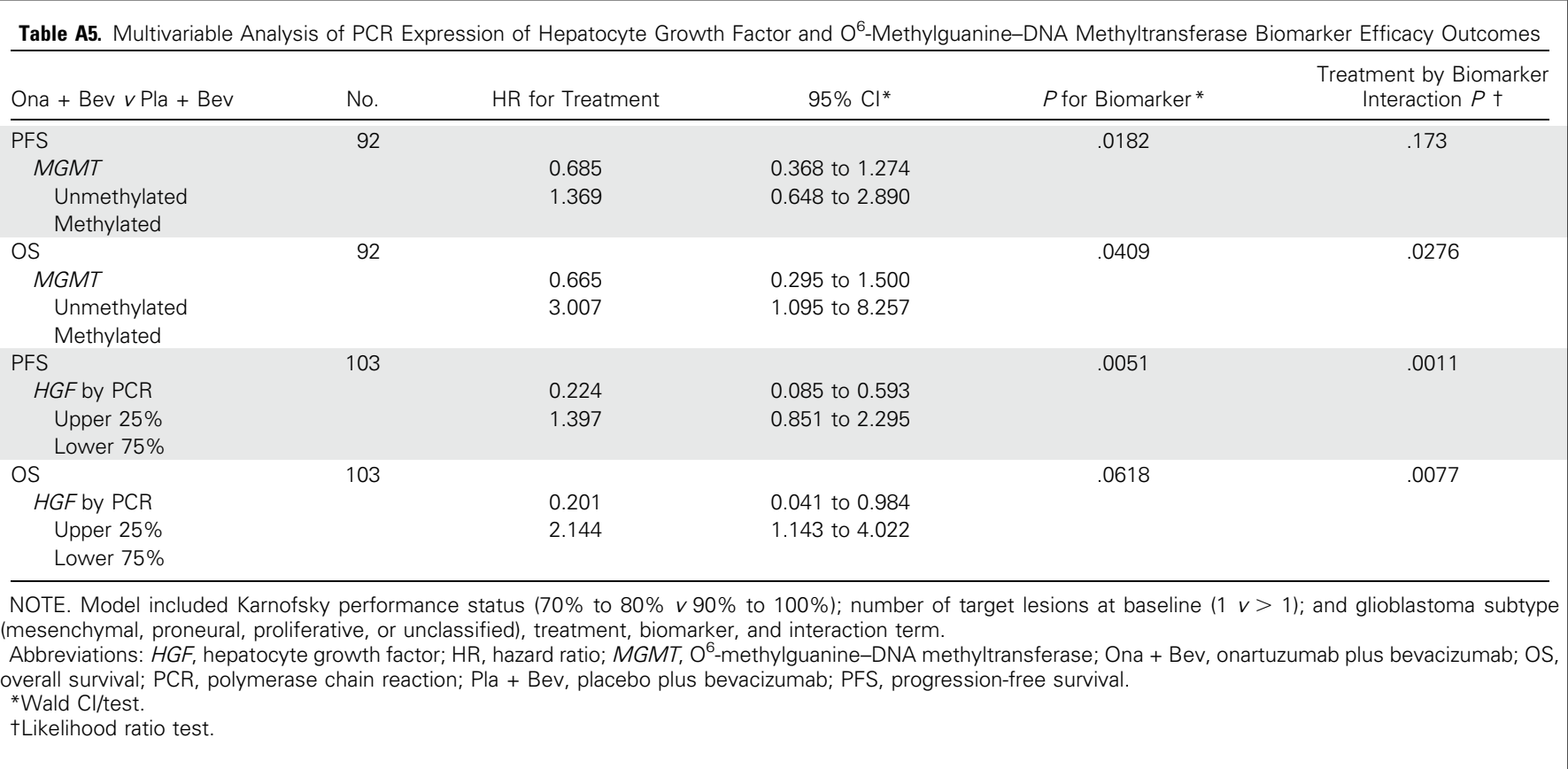

